\title{
Development and Validation of a Multi-Strand Solver for Complex Aerodynamic Flows
}

\author{
Vinod K. Lakshminarayan ${ }^{*, a, 1}$, Jayanarayanan Sitaraman ${ }^{\mathrm{b}, 1}$, Beatrice Roget ${ }^{\mathrm{a}, 1}$, Andrew M. Wissink ${ }^{\mathrm{c}, 2}$ \\ ${ }^{a}$ Science $\mathcal{E}$ Technology Corporation, NASA Ames Research Center, Moffett Field, CA \\ ${ }^{b}$ Parallel Geometric Algorithms LLC, Sunnyvale, CA \\ ${ }^{c}$ US Army Aviation Development Directorate - ADD (AMRDEC), Moffett Field, CA
}

\begin{abstract}
The strand grid approach is a flow solution method where a prismatic-like grid using "strands" is grown to a short distance from the body surface to capture the viscous boundary layer and the rest of the domain is covered using an adaptive Cartesian grid. The approach offers several advantages in terms of nearly automatic grid generation and adaptation, ability to implement fast and efficient flow solvers that use structured data in both the strand and Cartesian grids, and the development of efficient and highly scalable domain connectivity algorithm. An improvement to this approach is the multi-strand strategy, where multiple strands are allowed from each surface vertex to enhance grid resolution near sharp corners. This paper introduces a fully parallel and highly efficient flow solver called mStrand that is developed from ground-up to operate on multi-strand meshes. The strand solver is integrated to HPCMP CREATE $^{\mathrm{TM}}-\mathrm{AV}$ Helios framework to simulate complex aerodynamic flows. Detailed validation of the solver is shown on problems with varying degrees of complexity and comparison with experimental data. A performance study shows that the strand solver is nearly as efficient as a structured grid solver.
\end{abstract}

Key words: strand grid, multi-strand approach, compressible flow, finite volume method, dual-mesh paradigm,

Cartesian AMR, hovering rotor, tip vortices

\section{Introduction}

The use of computational fluid dynamics (CFD) arise in many scientific and engineering applications. One of the most notable difficulties in applying CFD early in the design process is the lack of automation in mesh generation. With the advent of High Performance Computing (HPC), the fraction of time required for mesh generation and prob-

\footnotetext{
${ }^{*}$ Corresponding author

Email addresses: vinod.k.lakshminarayan.ctr@mail.mil (Vinod K. Lakshminarayan), jayanarayanan.sitaraman.ctr@mail.mil (Jayanarayanan Sitaraman), beatrice.f.roget.ctr@mail.mil (Beatrice Roget), andrew.m.wissink.civ@mail.mil (Andrew M. Wissink)

${ }^{1}$ Research Scientist

${ }^{2}$ Aerospace Engineer

Preprint submitted to Computers $\mathcal{E}$ Fluids

December 15, 2016
} 
lem setup has increased significantly. Automating this process, coupled with efficient parallel algorithms, will enable turnaround times that are appropriate for engineering design.

Traditionally, there are two primary types of flow solvers available - structured grid solvers and unstructured grid solvers. Structured grid solvers are efficient and allow use of higher order algorithms. The efficiency of structured grid solvers enables use of many more grid points to provide accurate solution. However, generating structured grid, even while using multi-block structured and/or overset meshes, can be extremely challenging. On the other hand, unstructured codes generally offer more flexibility and automation in grid generation and problem setup for geometrically-complex bodies such as fuselages and hubs. But, the overall computational cost associated with such solvers to provide comparative level of accuracy as a structured grid solver is significantly higher. Therefore, in order to perform high-fidelity modeling and simulation that has potential for very high degrees of automation, an approach is needed that achieves efficiency comparable to structured grid solvers but with a more automated mesh generation strategy.

One such approach is the strand grid approach $[1,2]$. In this approach, the volume mesh generation process is fully automated by extending a viscous-quality prismatic mesh directly from a surface tessellation composed of triangles, quadrilaterals, or other shapes. A near-body volume grid that resolves the viscous boundary layer is constructed by inflating the surface grid along curves that can be represented with few parametric quantities. These curves, referred to as "strands", are most commonly just straight lines, each represented by a pointing vector and a length (see Fig. 1). Strands extrude a short distance from the solid boundary and are clipped with a "clipping index", when intersected with the adaptive Cartesian grids, which cover the rest of the domain to the outer boundaries.

Strand near-body meshes combined with Cartesian off-body grids not only support automatic viscous mesh generation and adaptation but also provide a number of other advantages. First, both strand and Cartesian meshes possess some grid structure, facilitating efficient implementations of high-order accurate discretizations and solution methods such as high-order finite differencing, line-implicit solvers, and directional multi-grid coarsening. Second, each strand on the strand mesh can be represented with minimal information such as strand direction, length etc., thus reducing the entire volume mesh description to primarily a surface definition. This allows use of highly efficient and highly scalable domain connectivity package [3] to facilitate data exchange between the two mesh types. Third, both the strand and Cartesian grids easily permit use of Adaptive Mesh Refinement (AMR) enabling local refinement around important flow features. Because the strand grid is structured in the normal direction, unstructured refinement is performed only on the surface which avoids many of the cell quality issues that often occur with frequent and persistent adaptation of 3D tetrahedral elements.

Although a strand-based framework offers a potential solution for complete automation, a robust strand solver that 


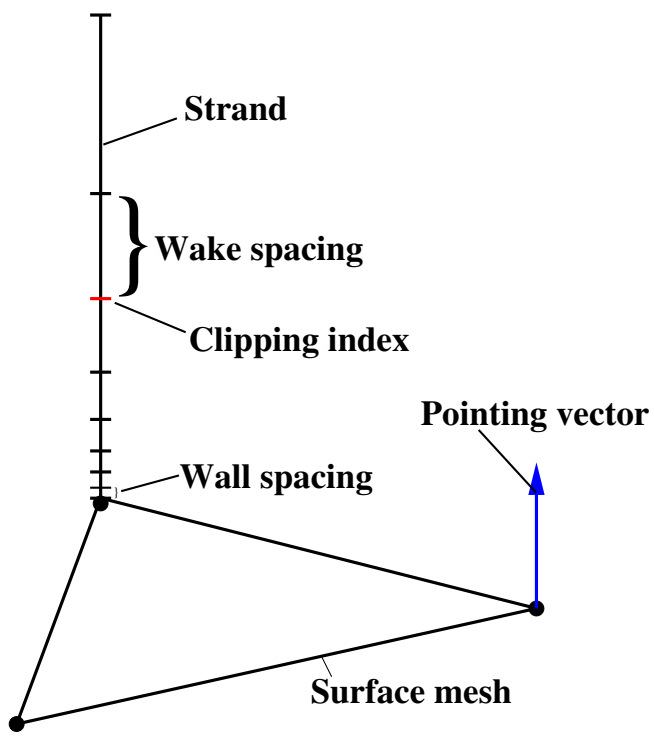

Figure 1: Strand pointing vector and clipping index.

can simulate realistic engineering problems has not yet been realized. From the several research articles published [4, $2,5,6,7,8]$ in the past, it is seen that one of the primary challenges in simulating complex geometries with this framework arises when handling convex corners. With a single strand per surface vertex, the regions near convex corners are not well resolved, which results in poor solver convergence and accuracy. The use of multiple strands allows better resolution of these corners, but the grid can have extremely small volumes that can affect the convergence of the flow solver. Furthermore, surface tessellations with concave-convex type edges/corners can pose scenarios where a single-strand direction cannot yield a valid volume grid. Having multiple strand provides an elegant solution to this problem. Generation of multi-strand type meshes has been explored in detail by Haimes et al. [9]. In this work, a multi-strand (mStrand) solver is developed from ground up to be able to handle multiple strands emanating from the surface as well as small volumes resulting from such a mesh. The solver is implemented to be fully parallel from its inception and particular emphasis is made on its robustness and efficiency.

As a part of this work, mStrand is integrated with the Helios framework to simulate complex aerodynamic problems. The Helios software $[10,11,12]$ is a high-fidelity rotary-wing product of the HPCMP CREATE ${ }^{\mathrm{TM}}-\mathrm{AV}_{\text {(air }}$ vehicles) program [13]. The code was introduced in 2010 to provide a high-fidelity analysis capability to the DoD for the acquisition of new rotary-wing aircraft and is now in routine use at several DoD sites and US Helicopter companies. Helios uses a dual mesh paradigm [14] as the basis of the CFD aerodynamics solution procedure, where a near-body solver is used to capture viscous flow around complex geometry, and block structured Cartesian solver [15] in the off-body to resolve the wake through a combination of high-order algorithms and adaptive mesh refinement 
(AMR). The PUNDIT [16] domain connectivity software facilitates parallel data exchange between the two mesh types as well as enables relative motion between the mesh systems. Coordination of the different codes is managed through a lightweight and flexible Python-based infrastructure [17, 18]. Once fully tested, mStrand is intended to become one of the primary near-body solver in the Helios framework.

The remainder of the paper is organized as follows. Section 2 provides a detailed description of mStrand. Section 3 performs accuracy analysis of the flow solver. This is then followed by a description of the mesh generation strategies used in this work in Section 4. Detailed validation of mStrand in both standalone mode and as part of Helios framework is provided in Section 5. A preliminary performance analysis of mStrand is performed in Section 6, where the strand solver is compared with the performance of established unstructured and structured grid solvers. Concluding remarks are offered in Sec. 7.

\section{Multi-Strand (mStrand) Flow Solver}

\subsection{Governing Equations}

The Reynolds-averaged Navier-Stokes (RANS) equations in a general moving coordinate system in three dimensions is solved in mStrand. Turbulence closure is accomplished with the Spalart-Allmaras (SA) model[19]. The RANS-SA equations may be expressed as

$$
\frac{\partial Q}{\partial t}+\frac{\partial F_{j}}{\partial x_{j}}-\frac{\partial F_{j}^{v}}{\partial x_{j}}=S
$$

where the conserved variables, $Q$, inviscid fluxes, $F_{j}$, viscous fluxes, $F_{j}^{v}$, and source term, $S$, are defined as

$$
\begin{gathered}
Q=\left(\begin{array}{c}
\rho \\
\rho u_{i} \\
\rho e \\
\rho \tilde{v}
\end{array}\right), \quad F_{j}=\left(\begin{array}{c}
\rho\left(u_{j}-u_{j}^{b}\right) \\
\rho u_{i}\left(u_{j}-u_{j}^{b}\right)+p \delta_{i j} \\
\rho e\left(u_{j}-u_{j}^{b}\right)+p u_{j} \\
\rho \tilde{v}\left(u_{j}-u_{j}^{b}\right)
\end{array}\right), \\
0 \\
F_{j}^{v}=\left(\begin{array}{c}
0 \\
0 \\
\sigma_{i j} \\
\sigma_{i j} u_{i}-q_{j} \\
\frac{\eta}{\sigma} \frac{\partial \tilde{v}}{\partial x_{j}}
\end{array}\right), \quad S=\left(\begin{array}{c} 
\\
\mathcal{P}-\mathcal{D}+C_{b 2} \rho \frac{\partial \tilde{v}}{\partial x_{k}} \frac{\partial \tilde{v}}{\partial x_{k}}
\end{array}\right) .
\end{gathered}
$$

Here, $\rho$ is the density, $u_{i}$ is the Cartesian velocity vector, $u_{i}^{b}$ is the mesh velocity vector, $e$ is the total energy per unit mass, $\tilde{v}$ is the turbulence working variable, $p$ is the pressure, $\sigma_{i j}$ is the deviatoric stress tensor, $q_{j}$ is the heat flux 
vector, and $\eta / \sigma$ is the turbulent diffusion coefficient. The turbulent source term consists of a production term, $\mathcal{P}$, and a destruction term $\mathcal{D}$. The stress tensor is defined as

$$
\sigma_{i j}=2\left(\mu+\mu_{T}\right) s_{i j},
$$

where $\mu$ is the dynamic viscosity, $\mu_{T}$ is the turbulent viscosity, and $s_{i j}$ is the rate of strain tensor, defined as

$$
s_{i j}=\frac{1}{2}\left(\frac{\partial u_{i}}{\partial x_{j}}+\frac{\partial u_{j}}{\partial x_{i}}\right)-\frac{1}{3} \frac{\partial u_{k}}{\partial x_{k}} \delta_{i j}
$$

The heat flux vector is obtained with Fourier's Law,

$$
q_{j}=-C_{p}\left(\frac{\mu}{P r}+\frac{\mu_{T}}{P r_{T}}\right) \frac{\partial T}{\partial x_{j}}
$$

where $C_{p}$ is the specific heat, $\operatorname{Pr}$ is the Prandtl number, $P r_{T}$ is the turbulent Prandtl number, and $T$ is the temperature. The ideal gas equation of state, $p=\rho R T$ is used to close the equations.

The standard SA model is used when the turbulent working variable is positive. Details of the positive model, including the well-known definitions of the production and destruction terms, may be found in the original work by Spalart and Allmaras [19]. Modifications to the model to accommodate negative values of the turbulence working variable have been suggested recently by Allmaras [20] and are employed in this work. In the case of negative values of $\tilde{v}$, the following turbulence equation replaces the standard model:

$$
\frac{\partial \tilde{v}}{\partial t}+\left(u_{j}-u_{j}^{b}\right) \frac{\partial \tilde{v}}{\partial x_{j}}=C_{b 1}\left(1-C_{t 3}\right) \Omega \tilde{v}+C_{w 1}\left(\frac{\tilde{v}}{d}\right)^{2}+\frac{1}{\sigma}\left[\frac{\partial}{\partial x_{j}}\left(\left(v+\tilde{v} f_{n}\right) \frac{\partial \tilde{v}}{\partial x_{j}}\right)+C_{b 2} \frac{\partial \tilde{v}}{\partial x_{k}} \frac{\partial \tilde{v}}{\partial x_{k}}\right]
$$

where,

$$
f_{n}=\frac{C_{n 1}+\chi^{3}}{C_{n 1}-\chi^{3}}, \quad\left(C_{n 1}=16\right) .
$$

Here, $\Omega$ is the vorticity magnitude, $d$ is the distance to the nearest wall, and $\chi=\tilde{v} / v$ is the ratio of the turbulent working variable to the kinematic viscosity of the fluid. All other constants in Equation 6 take the values found in the standard model.

\subsection{Solution Methodology}

The strand grid spatial discretization is based on a vertex-centered finite volume approach, where a dual-cell is constructed around each grid point. The strand solver accommodates both quadrilateral and triangular prisms depending on the surface topology and can handle general prismatic mesh in the normal (strand) direction. Each 


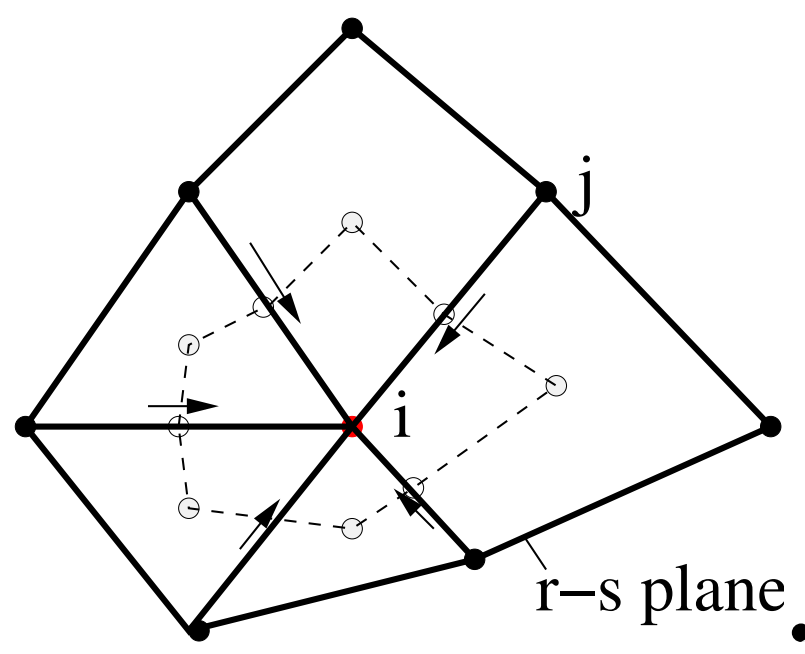

(a) Surface plane

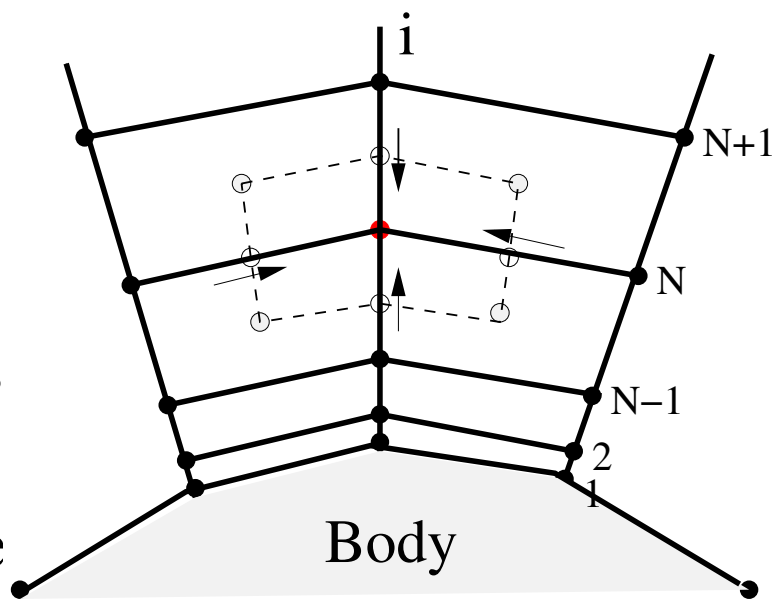

(b) Strand direction

Figure 2: Projection of dual-cell control volume on surface plane and strand direction.

vertex in the volume grid is represented by an index pair $(i, N)$, where $i$ is the surface index and $N$ is the strand-layer index. Figure 2 shows projections of the median dual cell constructed around vertex $(i, N)$ on the surface (referred as $r-s)$ plane and the strand direction. Also shown are the control volume interfaces where the fluxes are evaluated. The total flux on any vertex is obtained as a sum of contribution from all its connected edges.

\subsubsection{Face Center, Cell Centroid and Dual-Cell Volume}

The first step in the solution methodology is to compute the face centers and primal cell centroids to form the dual-cells and calculate the dual-cell volumes. The face centers of triangular and quadrilateral faces are estimated as:

$$
\vec{r}_{f c}=\frac{1}{N_{v}} \sum_{n=1}^{N_{v}} \vec{r}_{n},
$$

where $N_{v}$ is the number of vertices on the considered face ( 3 for triangles and 4 for quadrilaterals), $\vec{r}_{n}$ is the position vector of vertex $n$.

The primal cell centroid is computed using gradient theorem. The gradient theorem states that

$$
\int_{\Omega} \vec{\nabla} \varphi d V=\oint_{S} \varphi \vec{n} d S .
$$

Choosing $\varphi$ as $\frac{1}{2} \vec{r} \cdot \vec{r}=\frac{1}{2}\left(x^{2}+y^{2}+z^{2}\right)$, where the position vector $\vec{r}=x \hat{i}+y \hat{j}+z \hat{k}$, the cell centroid for any arbitrary 
polygon can be estimated as:

$$
\vec{r}_{c c}=\frac{1}{2 \Omega} \oint_{S}\left(x^{2}+y^{2}+z^{2}\right) \vec{n} d S=\frac{1}{2 \Omega} \sum_{i=1}^{N_{f}} \int_{S}\left(x_{i}^{2}+y_{i}^{2}+z_{i}^{2}\right) \vec{n}_{i} d S,
$$

which is essentially a surface integration over the $N_{f}$ faces of the polygon. Here, $\Omega$ is the primal cell volume, $\vec{n}_{i}$ is the face normal and $\vec{r}$ is the position vector. The surface integration on each face is performed using Gaussian-quadrature rule. For a quadratic function, the exact integral on triangles can be calculated using 3 quadrature points and that on bilinear quadrilaterals can be computed using 4 quadrature points.

Calculation of volume of an arbitrary polygon can also be reduced to a surface integration problem using the divergence theorem,

$$
\int_{\Omega} \vec{\nabla} \cdot \vec{\phi} d V=\oint_{S} \vec{\phi} \cdot \vec{n} d S
$$

Choosing $\vec{\phi}$ as the position vector, $\vec{r}$, the integral becomes:

$$
3 \Omega=\oint_{S} \vec{r} \cdot \vec{n} d S=\sum_{i=1}^{N_{f}} \int_{S} \overrightarrow{r_{i}} \cdot \overrightarrow{n_{i}} d S
$$

Thus,

$$
\Omega=\frac{1}{3} \sum_{i=1}^{N} \int_{S}^{\text {faces }} \overrightarrow{r_{i}} \cdot \overrightarrow{n_{i}} d S .
$$

For triangles and bilinear quadrilaterals, the face integral can be proved to be:

$$
\int_{S} \overrightarrow{r_{i}} \cdot \overrightarrow{n_{i}} d S=\vec{r}_{f c} \cdot \vec{A}_{f}
$$

where $\vec{r}_{f c}$ is face center defined in eqn. 7 and $\vec{A}_{f}$ is the face area.

\subsubsection{Inviscid Discretization}

The inviscid terms are computed using upwind formulation based on the approximate Riemann solver of Roe [21]. The inviscid flux associated with a particular control interface is given by:

$$
\hat{\mathcal{F}}=\frac{1}{2}\left(\mathcal{F}\left(Q_{R}\right)+\mathcal{F}\left(Q_{L}\right)\right)-\frac{1}{2}\left|\hat{A}\left(Q_{R}, Q_{L}\right)\right|\left(Q_{R}-Q_{L}\right),
$$


where $Q_{L}$ and $Q_{R}$ represent the value of the flow variables at the left and right sides of the control volume interface, $\mathcal{F}=F_{j} n_{j}$ is the directed flux at the control volume interface with normal $n_{j}$, and $\hat{A}$ is the directed Roe-averaged Jacobian. $\hat{A}$ has a dependency on the grid velocity at the control interface for a non-stationary mesh. To satisfy Geometric Conservation Law [22] (GCL), the grid velocity is computed as the ratio of volume swept by the control face to the face area. To obtain second-order spatial accuracy, the left and right states are obtained by extrapolating the control volume values to the control volume interface. For example, $Q_{L}$ corresponding to any edge $e$ associated with the vertex $(i, N)$ can be written as:

$$
Q_{L}=Q_{(i, N)}+\phi_{(i, N)}^{e}\left(\frac{1}{3} \nabla_{f} Q_{(i, N)}^{e}+\frac{1}{6} \nabla_{b} Q_{(i, N)}^{e}\right)
$$

where $Q_{(i, N)}$ is the value of flow variable in the control volume $(i, N), \nabla_{f} Q_{(i, N)}^{e}$ and $\nabla_{b} Q_{(i, N)}^{e}$ are an estimate of forward and backward gradients at the vertex $(i, N)$ along the edge $e$ and $\phi_{(i, N)}^{e}$ is the gradient limiter computed for each flow variable. In this work, a differentiable form of Van-Albada's limiter [23] is used and is given by:

$$
\phi_{(i, N)}^{e}=\frac{2 \nabla_{f} Q_{(i, N)}^{e} \nabla_{b} Q_{(i, N)}^{e}+\epsilon}{\left(\nabla_{f} Q_{(i, N)}^{e}-\nabla_{b} Q_{(i, N)}^{e}\right)^{2}+2 \nabla_{f} Q_{(i, N)}^{e} \nabla_{b} Q_{(i, N)}^{e}+\epsilon},
$$

where, $\epsilon$ is a small number used to prevent division by zero. Other limiter options such as Koren's differentiable limiter [24] and Venkatakrishnan multi-dimensional limiter [25] are available in the solver. For an edge connected by the vertices in the $r$-s plane, such as one connecting the vertices $(i, N)$ and $(j, N)$ (see Fig.2(a)), the forward and backward gradients at the vertex $(i, N)$ along the edge $e$ are obtained as:

$$
\begin{aligned}
& \nabla_{f} Q_{(i, N)}^{e}=\frac{Q_{(j, N)}-Q_{(i, N)}}{\left|\vec{r}_{(j, N)}-\vec{r}_{(i, N)}\right|} \\
& \nabla_{b} Q_{(i, N)}^{e}=2 \vec{\nabla} Q_{(i, N)} \cdot \frac{\vec{r}_{(j, N)}-\vec{r}_{(i, N)}}{\left|\vec{r}_{(j, N)}-\vec{r}_{(i, N)}\right|}-\nabla_{f} Q_{(i, N)}^{e},
\end{aligned}
$$

where, $\vec{\nabla} Q_{(i, N)}$ is the full gradient of the flow solution computed at the vertex $(i, N)$ using either Green-Gauss or weighted least-squares method [26]. In the strand direction, the edge connecting vertices $(i, N)$ and $(i, N+1)$ is considered and the equivalent expression for the forward and backwards gradients can be written as:

$$
\begin{aligned}
& \nabla_{f} Q_{(i, N)}^{e}=\frac{Q_{(i, N+1)}-Q_{(i, N)}}{\left|\vec{r}_{(i, N+1)}-\vec{r}_{(i, N)}\right|} \\
& \nabla_{b} Q_{(i, N)}^{e}=\frac{Q_{(i, N)}-Q_{(i, N-1)}}{\left|\vec{r}_{(i, N)}-\vec{r}_{(i, N-1)}\right|},
\end{aligned}
$$


which essentially is a one-dimensional finite difference formula. The resulting inviscid normal (strand-direction) fluxes are formally second order accurate. However, on uniform meshes, they are third-order accurate if the solution is smooth.

\subsubsection{Viscous Discretization}

Computation of viscous flux requires solution and its gradient at the control volume interface. The interfacial gradient, directed along the considered edge, are obtained using a hybrid approach, which includes both the nodal gradient as well as the edge-based gradient. The hybrid approach avoids the odd/even decoupling artifact that is produced when a simple average of the gradient is used. The resulting expression is given by:

$$
\overline{(\nabla Q)_{i j}}=\frac{1}{2}\left[(\vec{\nabla} Q)_{i}+(\vec{\nabla} Q)_{j}\right]-\frac{1}{2}\left[(\vec{\nabla} Q)_{i}+(\vec{\nabla} Q)_{j}\right] \cdot \frac{\overrightarrow{r_{j}}-\overrightarrow{r_{i}}}{\left|\overrightarrow{r_{j}}-\overrightarrow{r_{i}}\right|}+\frac{Q_{j}-Q_{i}}{\left|\overrightarrow{r_{j}}-\overrightarrow{r_{i}}\right|}
$$

Here, the vertices are represented by single indices $i$ and $j$ for simplicity. There is no difference in the calculation of above expression on an $r$-s edge or a normal edge. The nodal gradients computed for the inviscid flux computation are re-used here. The interfacial solution required for viscous flux computation is obtained as a simple average of the nodal solution. The resulting discretization provides second-order implementation of full Navier-Stokes terms.

\subsubsection{Turbulence Model Discretization}

At present, only a first-order discretization of the SA turbulence model equation is implemented. The authors found that use of high-order discretization for turbulence terms compromises the robustness of the flow solver. Future studies will focus on developing a stable high-order turbulence model implementation. The SA model also requires calculation of distance to the wall function at all vertices. In strand grids, a good approximation of this function can be obtained by measuring the distance from the first strand layer of the considered surface index.

\subsubsection{Boundary Conditions}

For vertex-centered codes, the boundary nodes lie precisely on the domain boundaries. As a result, the dual-cell around boundary nodes is closed using the boundary faces, see Fig. 3. A weak form of boundary conditions can be implemented using fluxes through these faces. Currently, four types of boundary conditions are supported in mStrand: 1) adiabatic inviscid wall, 2) adiabatic viscous wall, 3) symmetry bc, and 4) characteristic farfield bc. The inviscid and symmetry boundary fluxes are imposed as physical flux through the boundary face with zero normal velocity. The viscous wall boundary condition cannot be implemented just through boundary fluxes. The mass and energy boundary fluxes are set the same as that in the inviscid condition, but the momentum equations at the boundary nodes 


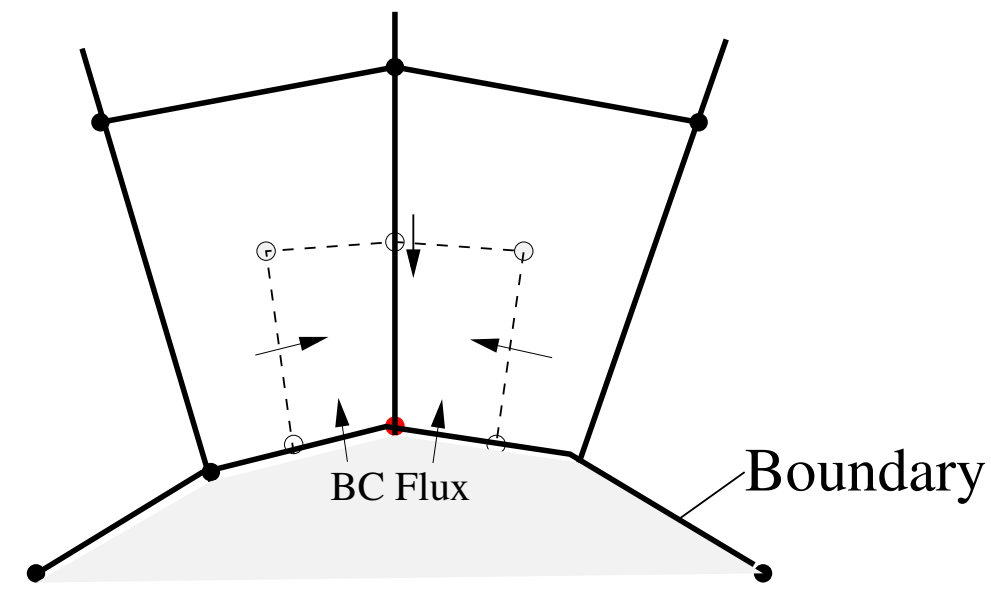

Figure 3: Dual-cell control volume near boundary.

are replaced to Dirichlet conditions, given by $u_{i}=u_{i}^{\text {wall }}$. Here $u_{i}^{\text {wall }}$ is the structural velocity at the wall. When the strand solver is used in isolation, a weak form of characteristic boundary condition is imposed at the farfield.

\subsubsection{Implicit Solution}

The spatial discretization of the inviscid, viscous and turbulence model fluxes along with the boundary conditions result in a large coupled set of ordinary differential equations, given by:

$$
V \frac{\partial Q}{\partial t}+R(Q)=0
$$

Here, $R(Q)$ is the spatial residual and $V$ is the cell volume. Adding a pseudo-time term to the discretized equations, we obtain:

$$
V \frac{\partial Q}{\partial \tau}+V \frac{\partial Q}{\partial t}+R(Q)=0
$$

where, $\tau$ is the pseudo-time variable. For steady problems, the time-derivative term is zero. For unsteady problems, second-order backward differencing (BDF2) is used to compute the temporal discretization. The pseudo-time derivative is approximated using backward Euler scheme. This allows to march the solution to a (pseudo-)steady state. In both steady and unsteady problems, the spatial discretization is evaluated at the new time level, leading to an implicit scheme. Since these quantities are not known explicitly, a linearization is performed at the current time level, leading to the following linear system to be solved at each pseudo-time step:

$$
\left[\frac{V}{\Delta \tau_{k}} I+\frac{\partial \hat{R}^{k}}{\partial Q}\right]\left(Q^{k+1}-Q^{k}\right)=-\hat{R}\left(Q^{k}\right)
$$


Here, $k$ is the iteration number, $\hat{R}(Q)$ is the total residual containing the spatial discretization along with the time derivative terms in an unsteady problem and $\partial \hat{R} / \partial Q$ is the Jacobian of the residual. The dual-time step size can be different for various vertices and can be unequal between iterations.

The linear system in Equation 22 is solved using a preconditioned matrix-free GMRES. Since GMRES [27] only requires the evaluation of matrix vector products of the form $\frac{\partial R}{\partial Q} \cdot \delta \vec{v}$, it is possible to not compute the Jacobian explicitly, and evaluate Jacobian vector product directly using Frèchet derivative, given by

$$
\frac{\partial \hat{R}}{\partial Q} \cdot \delta \vec{v}=\frac{\hat{R}(Q+\epsilon \delta \vec{v})-\hat{R}(Q)}{\epsilon}
$$

where $\epsilon$ is a small parameter. As is evident, this requires one additional residual evaluation for each matrix vector product.

GMRES is not an effective solver by itself for highly non-linear problems such as Navier-Stokes equations and requires a preconditioner to provide good convergence. The convergence of the linear solver has a direct correlation to the quality of the preconditioner. In this work, a specialized strand-based preconditioner is developed. The precondioner matrix is constructed using the first order Jacobian terms, retaining only the nearest neighbor contributions. The Jacobian matrix is fully coupled between mean-flow and turbulence equations. For any vertex $(i, N)$, the preconditioning system of linear equations can be written as:

$$
\begin{aligned}
\frac{\partial \hat{R}_{(i, N)}}{\partial Q_{(i, N-1)}} \Delta Q_{(i, N-1)}+\left[\frac{V}{\Delta \tau_{(i, N)}} I+\frac{\partial \hat{R}_{(i, N)}}{\partial Q_{(i, N)}}\right] \Delta Q_{(i, N)} & +\frac{\partial \hat{R}_{(i, N)}}{\partial Q_{(i, N+1)}} \Delta Q_{(i, N+1)} \\
& +\sum_{j \in \mathcal{N}(i)} \frac{\partial \hat{R}_{(i, N)}}{\partial Q_{(j, N)}} \Delta Q_{(j, N)}=-\hat{R}\left(Q_{(i, N)}\right),
\end{aligned}
$$

where $\mathcal{N}(i)$ is the neighborhood of surface index $i$ in the $r$-s plane. The above equation can be recast into

$$
\begin{aligned}
\frac{\partial \hat{R}_{(i, N)}}{\partial Q_{(i, N-1)}} \Delta Q_{(i, N-1)}+\left[\frac{V}{\Delta \tau_{(i, N)}} I+\frac{\partial \hat{R}_{(i, N)}}{\partial Q_{(i, N)}}\right] \Delta Q_{(i, N)} & +\frac{\partial \hat{R}_{(i, N)}}{\partial Q_{(i, N+1)}} \Delta Q_{(i, N+1)} \\
& =-\hat{R}\left(Q_{(i, N)}\right)-\sum_{j \in \mathcal{N}(i)} \frac{\partial \hat{R}_{(i, N)}}{\partial Q_{(j, N)}} \Delta Q_{(j, N)},
\end{aligned}
$$

to form a block-tridiagonal system of equations in the strand direction. In the above equation, the dependence on the $r$-s plane is solved iteratively using a colored Gauss-Seidel method, where the surface nodes are tagged with colors using a greedy algorithm such that no two connected vertices have identical color values. Multiple forward and backward sweeps of Gauss-Seidel iterations are performed in the order of the surface node coloring. The update on each surface index corresponds to a block-tridiagonal line solve along the strand direction that updates the solution at 


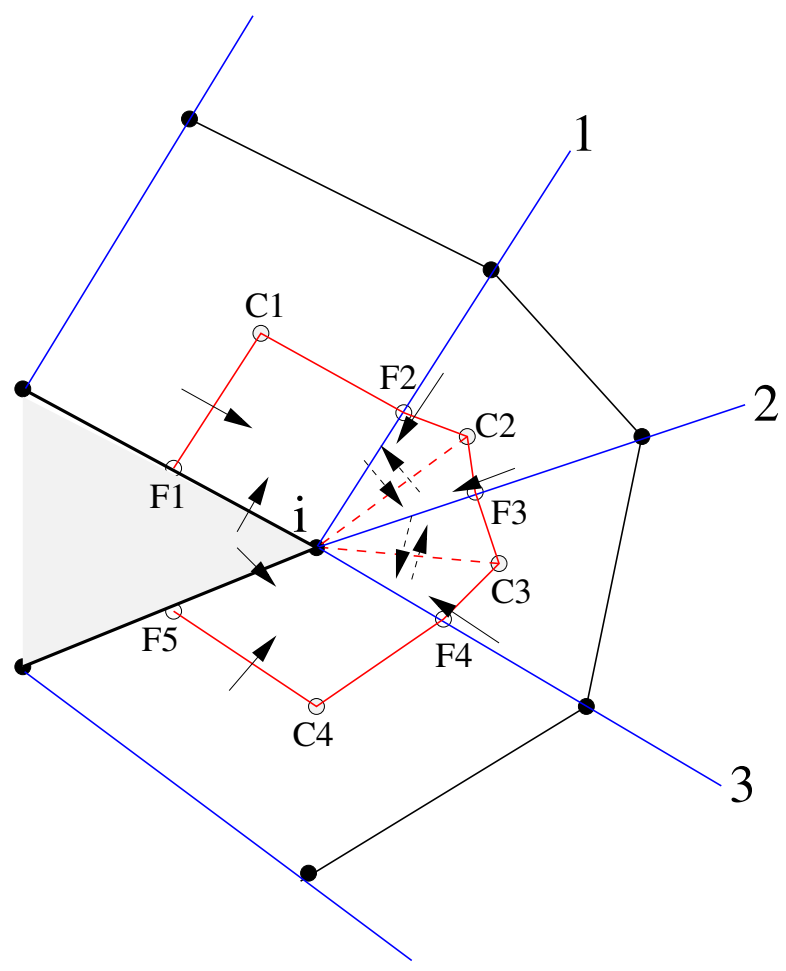

Figure 4: A schematic of control volume around a multi-strand node.

all vertices along the considered strand.

\subsection{Treatment of Multi-Strand Nodes}

As mentioned earlier, one of the key features of mStrand is its ability to handle multiple strands emanating from a surface node. Figure 4 shows a schematic of a surface vertex $(i, 1)$ that has multiple strands emanating from it. The schematic shows a 2D mesh, but the description provided in this section holds true as well in 3D. Since only a surface node is allowed to have multiple strands, a multi-strand node can be referred just using the surface index $i$, dropping the strand-layer index for brevity. There are three main challenges associated with using multiple strands - 1) The structure associated in the strand direction is no longer present in the first layer. The number of nodes and element connectivities on the first layer are different when compared to all other strand layers. This can lead to complex datastructures, degradation of the convergence of the linear solver and other challenges, 2) Several volume elements types including pyramids, tetrahedras, wedges and more are created at the first strand layer and the flow solver should be able to handle all the different element types, and 3) Improper treatment of multi-strand nodes can potentially lead to degradation of solver stability.

In mStrand, the first two challenges discussed above is addressed by a simple strategy of duplicating every multi- 
strand node into number of strands emanating from it. For example, in Fig. 4, the vertex $i$ is duplicated into three nodes that are coincident with each other. It is evident that this process immediately recovers the structure in all strand layers. The $r$-s plane nodes are indexed based on the inflated surface (defined later in Section 4). The body surface will have identical number of nodes, but some of these nodes can be coincident and the surface elements formed by those nodes may be degenerate. Using this strategy, the data structure for a multi-strand mesh is no different than a single strand mesh. Further, different volume element types are now represented as degenerate prisms or hexahedras. Eqns. 7 and 9 can be used directly to compute face centers and cell centroids to create dual-cells, thus allowing the solver to handle different element types. The above mentioned multi-strand strategy also allows the use of identical solution algorithms at all strand levels. This means each duplicated node owns a control volume and the fluxes are computed separately for each control volume. The fluxes are computed even along degenerate zero-length edges.

From a robustness point of view, it can be argued that if the solution algorithm used at a multi-strand node is consistent with any other node, the flow solution strategy will be the most stable. This means that the volume, total flux and flow solution gradients at multi-strand nodes should be computed in a consistent manner. Thus, the control volume around vertex $i$ should be defined by the median-dual curve $i-F 1-C 1-F 2-C 2-F 3-C 3-F 4-C 4-F 5-i$. However, only the surface connectivity information based on the inflated surface is available and this does not permit the construction of median-dual control volume around vertex $i$. Instead, the control volumes around the duplicated nodes (let us represent them as $i_{1}, i_{2}$ and $i_{3}$; the subscript the subscript corresponds to the strand number) can be constructed. The control volume around vertex $i_{1}$ is defined as $i-F 1-C 1-F 2-C 2-i$, and that for vertices $i_{2}$ and $i_{3}$ are $i-C 2-F 3-C 3-i$ and $i-C 3-F 4-C 4-F 5-i$, respectively. Evidently, the dual-cell volume of vertex $i$ is equal to the sum of dual-cell volumes of vertices $i_{1}, i_{2}$ and $i_{3}$. On a similar note, the fluxes at vertex $i$ cannot be calculated directly. Instead, the fluxes at vertices $i_{1}, i_{2}$ and $i_{3}$ can be computed separately. If started with identical flow solution and gradients at the duplicated nodes, the total flux associated with the vertex $i$ is equal to the sum of fluxes associated with vertices $i_{1}, i_{2}$ and $i_{3}$. Note that an equal and opposite flux goes from vertex $i_{1}$ to $i_{2}$ and back through the face $i-C 2$ and from vertex $i_{2}$ to $i_{3}$ and back through the face $i-C 3$. These fluxes cancel each other during the summation process. In a similar manner, it can be proved that the gradients at vertex $i$ can be calculated by aggregating appropriate quantities from its duplicated nodes. It can also be argued that each duplicated node of a multi-strand group represents the same region in the physical space and, therefore, should have identical flow solution at all time steps and they should be same as what would be computed at vertex $i$. The implementation strategy to obtain valid solution at all duplicated nodes of a multi-strand group involves assigning one of these nodes as the master and aggregating information from the slave nodes at appropriate locations. The aggregated information is then redistributed back to the slave nodes to proceed ahead with the solution methodology. 
In regard to the linear solver, GMRES uses the Frèchet derivative for the matrix-vector product and, therefore, does not need any change for multi-strand nodes. But the Gauss-Seidel iteration at multi-strand nodes are modified from eqn. 25 to:

$$
\begin{aligned}
& {\left[\frac{V}{\Delta \tau_{(i, 1)}} I+\frac{\partial \hat{R}_{(i, 1)}}{\partial Q_{(i, 1)}}\right] \Delta Q_{(i, 1)}+\frac{\partial \hat{R}_{(i, 1)}}{\partial Q_{(i, 2)}} \Delta Q_{(i, 2)}=-\hat{R}\left(Q_{(i, 1)}\right)-\sum_{j \in \mathcal{N}(i)} \frac{\partial \hat{R}_{(i, 1)}}{\partial Q_{(j, 1)}} \Delta Q_{(j, 1)}} \\
& \left.-\sum_{\substack{k \in \cup(N), l \in \mathcal{M}(i) \\
k \notin \mathcal{M}(i) \\
k \notin \mathcal{N}(i)}}\left(\frac{\partial \hat{R}_{(i, 1)}}{\partial Q_{(k, 1)}} \Delta Q_{(k, 1)}+\frac{\partial \hat{R}_{(i, 1)}}{\partial Q_{(k, 2)}} \Delta Q_{(k, 2)}\right)\right),
\end{aligned}
$$

where $\mathcal{M}(i)$ is the set of all vertices in the multi-strand group that the vertex $(i, 1)$ belongs to. The solution along the strand direction can still be updated using a block-tridiagonal solver. With sufficient Gauss-Seidel iterations, the solution at all duplicated nodes of a multi-strand group converge to an identical value. As an additional measure of safety, the solution updates at these nodes are averaged at the end of the preconditioner sweeps.

\subsection{Parallel Implementation}

A fully parallel implementation of mStrand is obtained by partitioning the inflated surface mesh into contiguous blocks on the basis of surface elements using Metis [28], an example is shown in Fig. 5(a). The above partitioning strategy offers two main advantages - 1) A two-dimensional partitioning operation is relatively inexpensive and can be easily performed during run time 2) Each strand belongs to the same processor and therefore, the line solver along the strand direction does not require any inter-processor communication; thus providing the most efficiency.

After partitioning, a surface node can be part of multiple-processors and a surface edge can be associated with one or two processors. Figure 5(b) shows a schematic where a surface vertex $i$ is part of three processors A, B and C. It is evident from the schematic that the net flux at vertex $i$ is obtained by adding contributions along edges that are located in different processors. Further, certain edges, for example edge $i j$, can be part of multiple processors and the flux computation through these edges are split across two processors. To facilitate accurate flow calculations, all nodes and edges are assigned their master processors. If a node or an edge is associated with multiple processors, both master and slave processors perform any local operations and the information is accumulated in the master processor. This is achieved by establishing node- and edge- based communication patterns, which allows gathering and redistribution of information between master and slave processors.

Since the partitioning is done based on the inflated surface and not the body surface, the duplicated nodes associated with a multi-strand group can be split across multiple processors. In this circumstance, the multi-strand group is assigned a local master node for each processor and the local master of its master processor is designated as the global 


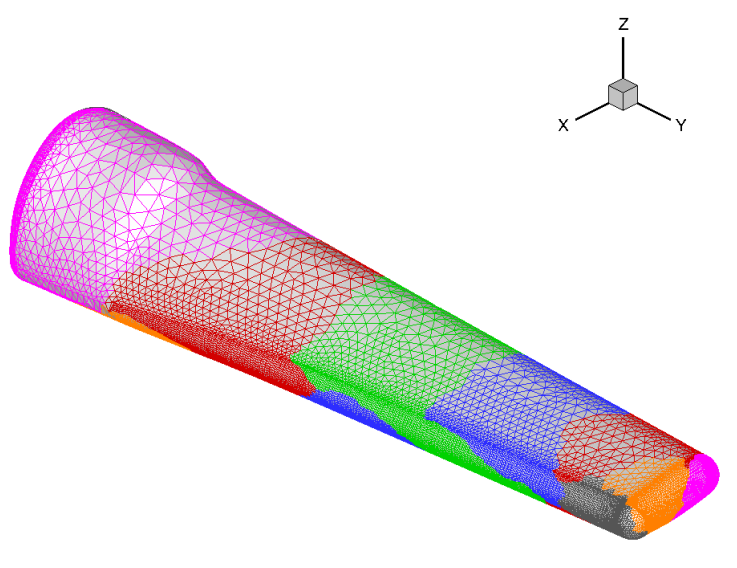

(a)

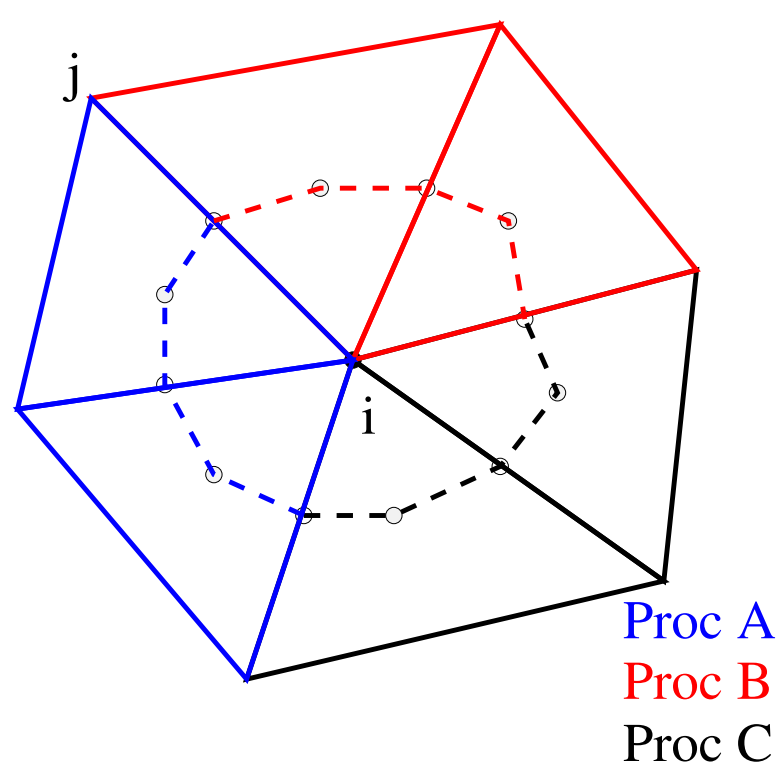

(b)

Figure 5: a) Partitioning of a blade mesh using Metis b) Schematic of a node in multiple processors.

master node. While gathering information at or from a multi-strand node, the information flows from local slave nodes to local master node and then to global master node through a multi-strand specific parallel communication. All node-based communication described in the previous paragraph is done after the multi-strand updates. The exact reverse path is followed while redistributing the information back to the slaves.

\section{Accuracy Analysis}

Prior to any flow simulation, the accuracy of mStrand is verified using the Method of Manufactured Solutions (MMS) [32, 33]. MMS is a procedure that can identify coding errors affecting the order of accuracy of a numerical method. With MMS, a "manufactured" solution is arbitrarily prescribed and substituted into the continuous equations to obtain an analytic source term. The discretized equations are then solved with the addition of the source term, added either as a point or as a volume weighted quantity, to obtain the discrete solution. The resulting solution can then be compared with the exact "manufactured" solution to obtain the solution error. A grid refinement study can then be performed to evaluate the formal order of accuracy of the method.

In the case of a complex set of equations, such as the Navier-Stokes equations, the exact solution must be carefully chosen so as to exercise all terms in the equation. The manufactured solution must also be carefully chosen such that it is not exactly satisfied by the discretized equation. The manufactured solution used for the current analysis is given by: 


$$
\begin{aligned}
& \rho(x, y, z)=\rho_{0}+\rho_{1} \exp \left(\frac{-x^{2}-y^{2}-z^{2}}{L^{2}}\right) \\
& u(x, y, z)=u_{1} \sin \left(\frac{x^{2}+y^{2}+z^{2}}{L^{2}}\right) \\
& v(x, y, z)=v_{1} \cos \left(\frac{x^{2}+y^{2}+z^{2}}{L^{2}}\right) \\
& w(x, y, z)=w_{1} \sin \left(\frac{x y+x z+y z}{L^{2}}\right) \\
& p(x, y, z)=p_{0}+p_{1} \sin \left(\frac{x}{L}\right) \sin \left(\frac{y}{L}\right) \sin \left(\frac{z}{L}\right),
\end{aligned}
$$

where $\rho_{0}$ is $1.0, p_{0}$ is $\frac{1}{\gamma}, \rho_{1}, u_{1}, v_{1}, w_{1}$ and $p_{1}$ are set to 0.1 and $L$ is the size of the domain, which is set to 1.0. The analytical source terms are determined using complex algebra techniques in Fortran90.

The MMS analysis is performed on spherical grids such as shown in Fig. 10(a) in Section 5.1. Spherical grids can be generated with ease and allows precise control of mesh spacing; at the same time, they provide sufficient complexity (grid curvature and stretching), that are necessary to provide rigorous code testing. The surface grid consists of triangular elements and the strand grid layers are stretched going from the inner to the outer boundary. The radius of the outer surface is set to unit length and the inner surface spans a fifth of this length. While computing the discrete solution, Dirichlet boundary condition equaling the exact manufactured solution is imposed at the inner and the outer surface.

Solution errors are computed by independently refining the surface and strand directions. The results obtained by employing both inviscid and viscous discretization terms are plotted in Fig. 6, which shows the error as a function of average grid spacing. In the plot, $\mathrm{N}_{\text {Surf }}$ is the number of surface nodes and $\mathrm{N}_{\mathrm{Norm}}$ is the number of vertices in the strand direction. For the error analysis along a particular direction (surface or strand), the grid spacing on the other direction is kept sufficiently fine to prevent any solution contamination due to discretization error in that direction. For the surface refinement, the number of surface elements is varied from 2000 to 200000 (1000 to 100000 surface nodes) with 400 strand layers. For the refinement in the strand direction, the number of strand layers is varied from 36 to 200, keeping the number of surface elements constant at 400000. From the plot, it is evident that the desired second order accuracy is achieved in both surface and strand refinements. The above analysis provides initial confidence in the flow solver. 


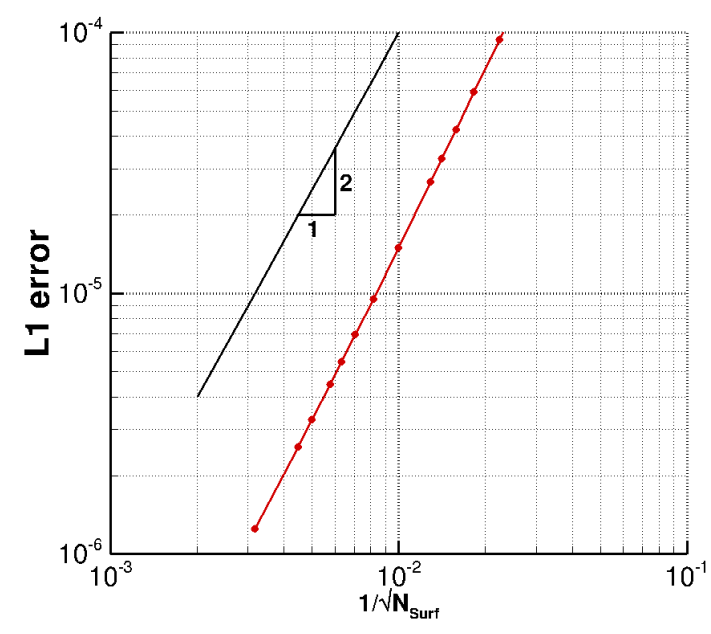

(a) Surface refinement

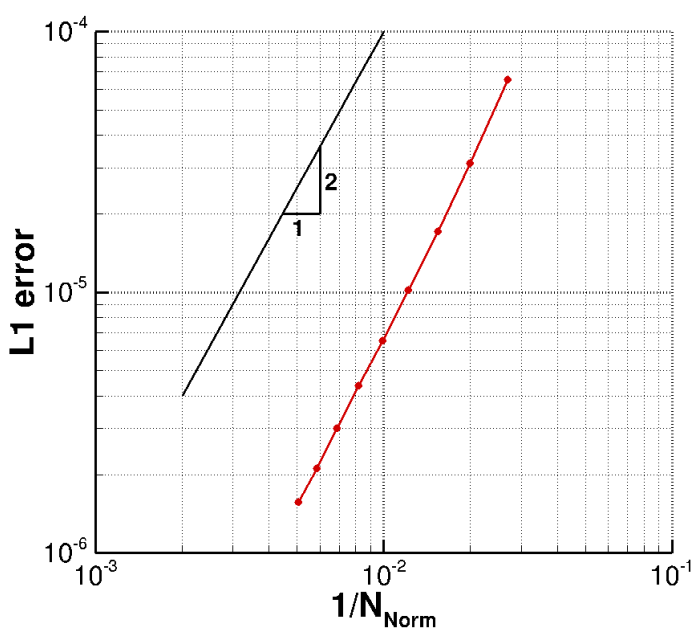

(b) Strand refinement

Figure 6: Solution error convergence using MMS analysis.

\section{Mesh Generation}

Unless otherwise stated, all the strand meshes used in this work are generated using the mStrandGen (ㅍulti- $\underline{\text { Strand }}$ Mesh Generator) code. The algorithm used in mStrandGen was first introduced in Ref. [34]. The inputs for the strand mesh generation are 1) a surface tessellation, which can be composed of triangles and quadrilaterals 2) a wall spacing to achieve a desired $y+$, a stretch ratio (typically $\sim 1.2$ ), and 3) a desired wake spacing. Strands are grown until the desired wake spacing, which is normally the finest spacing in the Cartesian grid used to resolve the wake, is achieved.

When intersected with the Cartesian grid, the strands are clipped with a "clipping index" associated with each strand (Fig. 1). Further, if the generated strands cross one another, which sometimes happen at concave corners, some elements become degenerate and are removed. This is again specified through the clipping index. The clipping index now defines the last valid prism element in the stack formed by strands.

While generating a strand mesh, first a single strand is grown from each surface vertex. The initial strand direction vector is constructed by computing the average face normals of the elements that share the vertex. All strands have same number of points and point distribution; so they grow to an identical length that depends on the input parameters. Spacing along each strand ranges from viscous at the root to a specified wake spacing at the extent, as shown in Fig. 1.

Next, smoothing is applied to improve coverage and mesh quality around sharp corners, such as the leading or trailing edges. A smoothing algorithm is applied which seeks to average each strand direction vector relative to its neighbors, effectively seeking isotropic spacing in the prism cells at the strand extents. Figure 7 shows a comparison of an unsmooothed and a smoothed mesh. Smoothing is implemented using an iterative scheme such that the degree of 
smoothing can be adjusted by the number of iterations. In general it is desirable to have a maximally smoothed strand mesh to yield isotropic cells at the strand extents that transition smoothly to the Cartesian off-body mesh. In practice, however, there are circumstances where too much smoothing produces negative volumes in the prism stack due to strand crossings. To avoid this issue, smoothing is applied in an iterative fashion whereby the maximal smoothing is applied that does not result in strand crossings. That is, after each smoothing iteration prism volumes are computed and smoothing is stopped if any negative volumes are found.

With a single strand per surface vertex, poor coverage can result in regions with highly convex corners, such as a trailing edge or flat tip (e.g. Fig. 7b). As mentioned earlier, this issue is alleviated by emanating multiple strands from a single surface vertex. The multi-strand procedure involves five steps, illustrated in Fig. 8; (a) build an adaptively tessellated iso-surface of a constant wall distance; (b) identify convex edges (CE) where surface face angles exceed a specified angle threshold $\left(40^{\circ}\right.$ was used in this work); (c) for surface indices not on the CE, find the point of minimum distance on the iso-surface, the line connecting these two points then defines the strand direction vector; (d) for surface indices that are on the CE, construct multiple strand directions based on face normal (e.g. point P in Fig. 8d has two strand directions, while point Q has three); (e) lastly, fill open areas with wedges and tetrahedra.

In some circumstances, the authors found that using a multi-level strand mesh generation strategy can improve the overall mesh quality. This strategy grows strands in multiple levels, typically two or three. The steps for generating the first level is exactly identical to that described above, but the strands are now grown only to a fraction of the specified final distance. In the subsequent levels, the tessellation of the inflated surface from the previous level is used as the starting point and strands are grown further outward. Only single strands are allowed at all levels other than the first one. The strands generated with this approach are not necessarily a straight line, but are made up of few line segments. However, the requirement of having a compact representation of the strands is still valid.

When mStrand is used within the Helios framework, the off-body Cartesian grid is generated following a Berger and Colella [29]-style multi-level block-structured AMR (SAMR) grid hierarchy. The coarsest level defines the physical extent of the computational domain and new levels are constructed from coarsest to finest. Each finer level is formed by selecting cells on the coarser level and then clustering the marked cells together to form block regions that will constitute the new finer level. The result is a hierarchy composed of nested refinement levels, with each level formed as a union of logically-rectangular grid regions.

The Cartesian grid is refined to match the spacing at the extents of the strand mesh, as demonstrated in Fig. 9. The location of the clip index elements $(x, y, z)$ and the wake spacing $\Delta s$ are provided to the Cartesian grid generator, cells on the Cartesian grid system that contain strand outer boundary elements are checked to see whether the Cartesian grid spacing $\Delta x$ is greater than the wake spacing $\Delta s$. If they are, $\Delta x>\Delta s$, the Cartesian cell is marked for refinement. All 


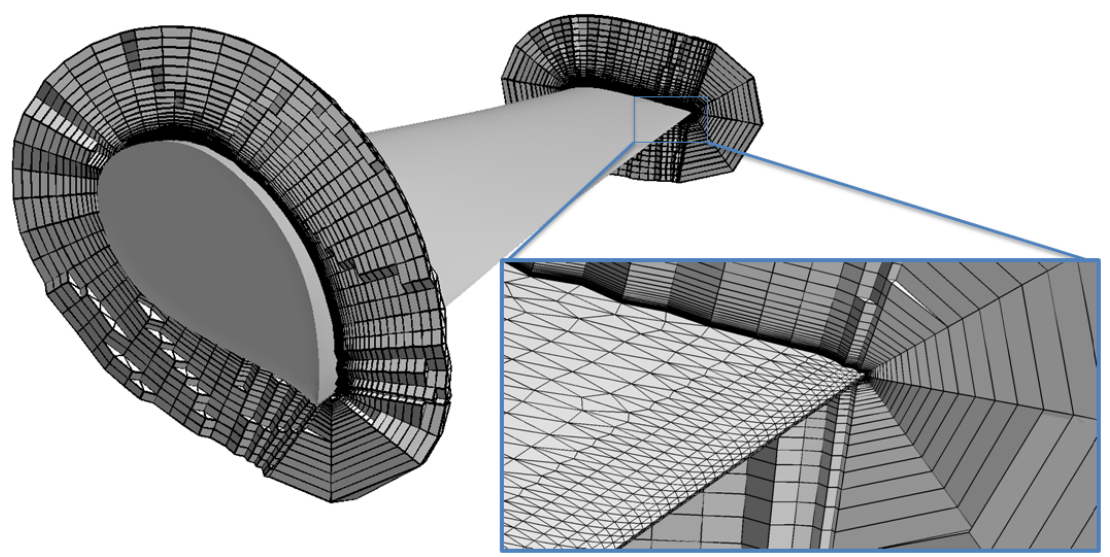

(a) Unsmoothed

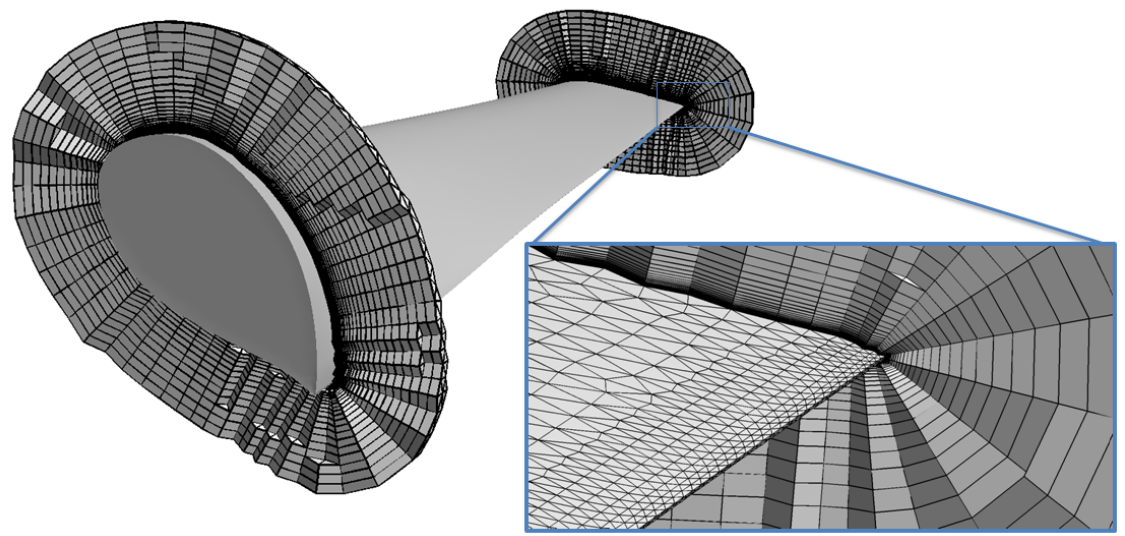

(b) Smoothed

Figure 7: Strand direction vector smoothing. (a) initial direction vector normal to surface, no smoothing applied, (b) strands with smoothed direction vectors. 


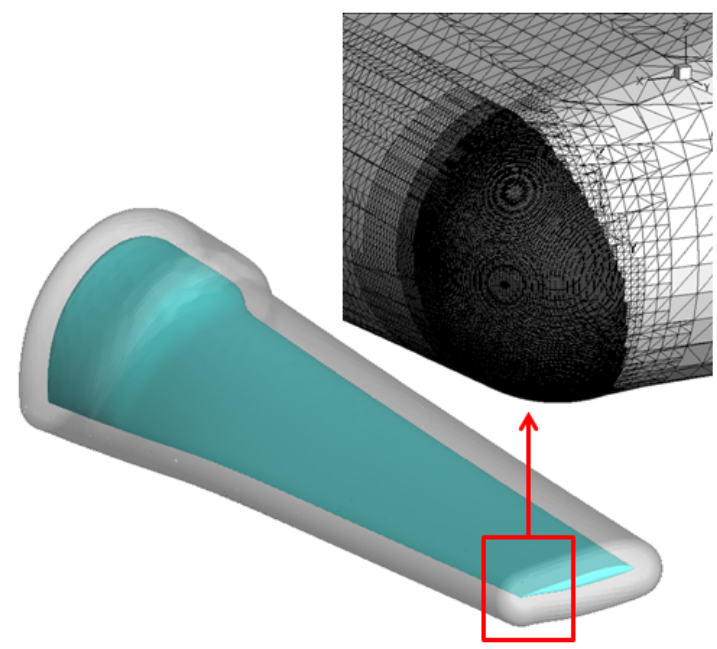

(a) Tessellated lifted iso-surface

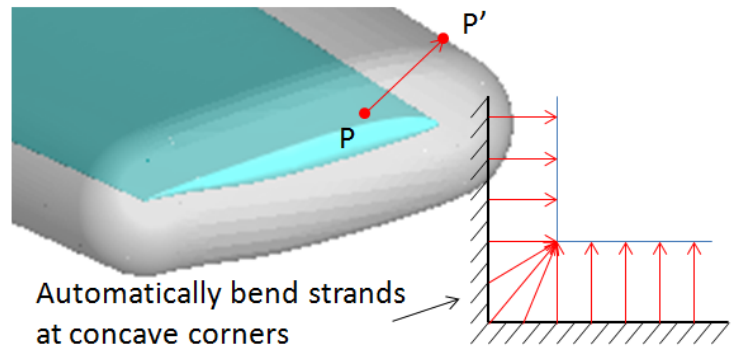

(c) Single strands - points not on CE

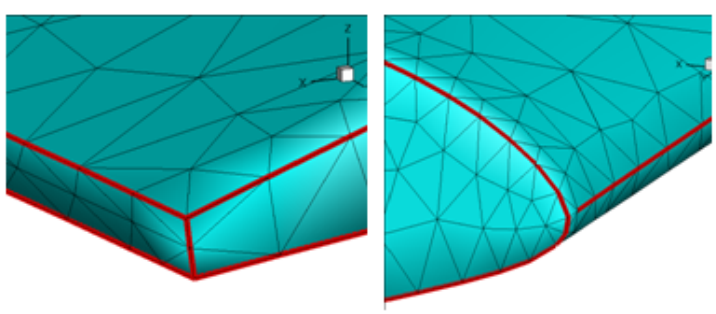

(b) Identify Convex Edges (CE)

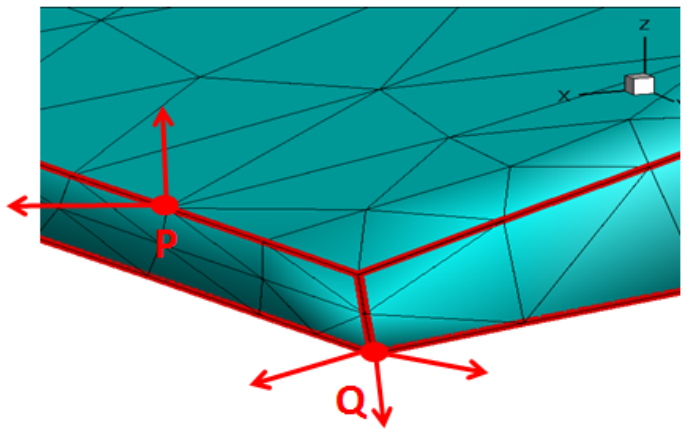

(d) Multiple strands - points on the CE

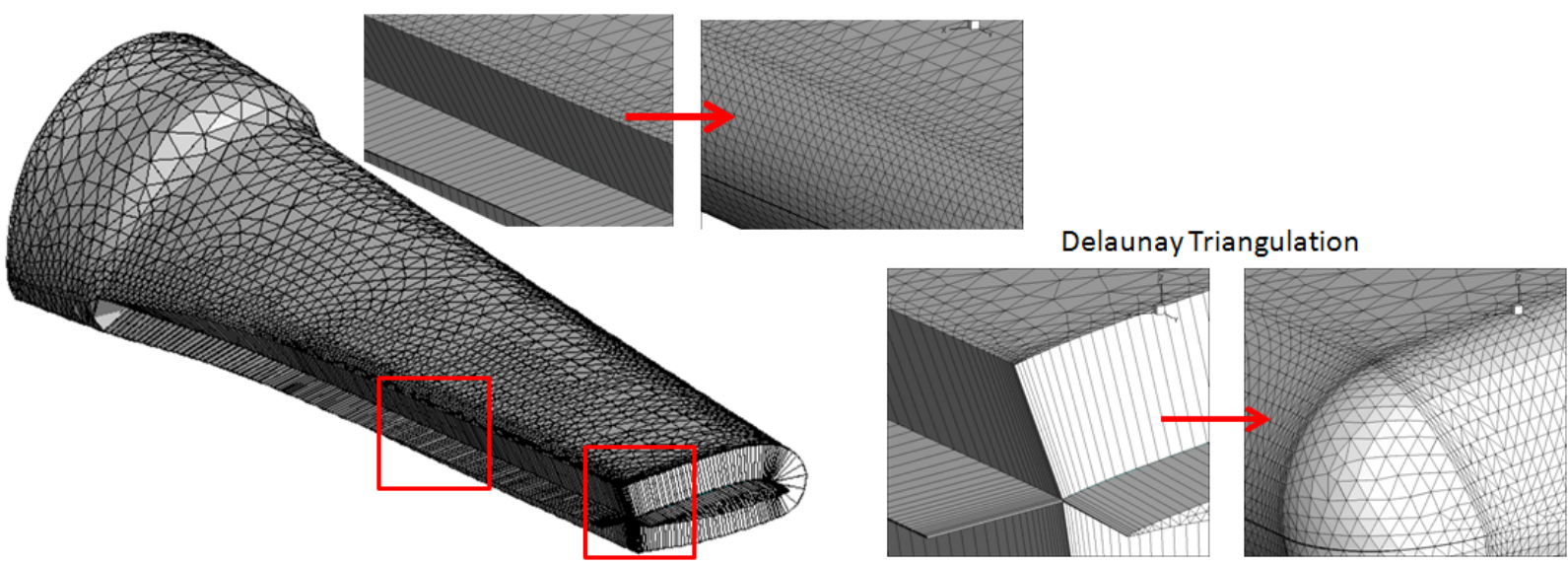

(e) Fill open region with wedges and tetrahedra

Figure 8: Multi-strand mesh generation: (a) build tessellated lifted surface (iso-surface of certain wall distance); (b) identify Convex Edges (CE) by comparing surface face angles; (c) form single strands from all vertices not lying on CE; (d) form multi-strand directions from vertices lying on CE; (e) fill in gaps with wedges and tetrahedra. 


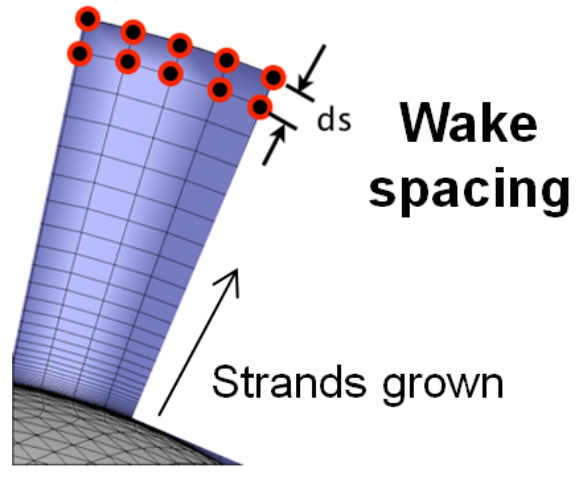

(a) Wake spacing at strand extents
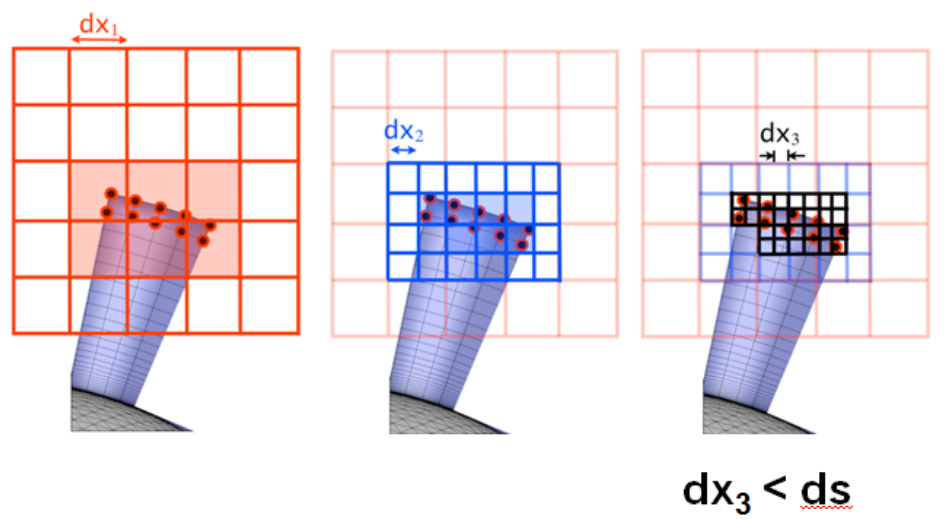

(b) Cartesian refinement to match spacing

Figure 9: Off-body adaptive Cartesian mesh generation to resolve overset interface between strand and Cartesian grids.

marked cells are clustered to construct a new finer level in the hierarchy. The process is repeated until no Cartesian cells are marked, meaning Cartesian blocks of equal or better spacing cover the entire set of strand grid extents, fully satisfying overset donor-receiver requirements and ensuring good mesh overlap at the Strand/Cartesian overset boundary.

After the initial generation of adaptive Cartesian grids to satisfy geometric requirements, grids are subsequently adapted throughout the simulation. Mesh cells that contain regions of swirling flow are identified using the scaled Qcriteria scheme proposed by Kamkar et al. [30]. Alternative solution quantities - e.g., vorticity or Q-criteria magnitude, density variation, entropy, etc. - could readily be substituted to drive refinement in the adaptive solution procedure. However, the scaled Q-criterion automates the wake refinement procedure, which is an advantage over other quantities. If a defined quantity such as vorticity or Q criteria is used, predicting beforehand the appropriate threshold is often difficult. Also, the strength of the vortex wake may be variable throughout the simulation. Multiple runs are therefore required to determine a suitable threshold quantity. The scaled Q quantity requires no such tuning and automatically adjusts to the changing scales of rotational flow in the wake.

\section{Validation}

This section provides a detailed validation of the mStrand solver. Several cases with varying degrees of complexity are considered. Laminar flow past sphere is used to validate the flow solver's inviscid and viscous term implementation. Turbulent flow past NACA0012 is used to verify the turbulence model implementation. Transonic flow past OneraM6 is used to demonstrate the capability of the solver to handle complex 3D problems and also to verify the interfacing of the solver within the Helios framework. A hovering rotor problem is simulated to exercise the moving 
mesh terms and to simulate highly complex flow field involving tip vortex formation and evolution. The final calculation showing TRAM rotor in hover is most complex case presented, composed of multiple bodies including three blades and a hub.

All Helios calculations use RANS/DES equation set in the Cartesian solver with fifth order spatial discretization for inviscid terms and fourth order spatial discretization for viscous terms. Simulations are performed using implicit BDF2 time integration for all time-dependent cases. The residual reported by mStrand represents a combined value of all the flow equations. The residual is computed using two steps - 1) L2-norm of each flow equation residual weighted by the inverse of control volume is computed separately and normalized by its value at the first time-step 2) the resulting five (or six, when turbulence equation is solved) values are combined and divided by the number of flow equations. This step re-normalizes the residual at the first time-step to one.

\subsection{Laminar Flow Past Sphere}

Laminar flow past sphere for a range of Reynolds number from 20 to 200 is used as the first case to validate $\mathrm{mStrand}$. The Mach number is set to 0.2 . Since the sphere does not have any convex corners, a multi-stranded mesh is not required for this problem. Further, the strand mesh can be extended all the way to farfield and therefore, the strand solver can be run and tested in isolation. These simulations are done using a mesh, shown in Fig. 10(a) having 8092 grid points on the surface, with 16180 triangular elements. 90 layers are used in the strand direction. Figure 10(b) shows the convergence of mStrand for four different Reynolds number. The residual is observed to converge 10 orders in all cases. The number of iterations required to converge increases with the Reynolds number. This behavior is observed in other solvers as well.

Figure 11 provides a qualitative comparison of the size of the separation bubble for four different Reynolds numbers with the results from Johnson and Patel [31]. As expected, the size of the separation bubble at convergence increases with Reynolds number. Furthermore, the solutions obtained are fully symmetric, providing confidence in the accuracy and validity of the solutions obtained. The locations of the center of the separation bubble, point of separation and the predicted drag coefficient are compared in Figure 12. All the flow features show good agreement with the results available in the literature.

\subsection{Turbulent Flow Past NACA0012 Airfoil}

To validate the turbulence model implementation of mStrand, turbulent flow past NACA0012 airfoil is considered. The details of the test case is provided on the NASA Langley turbulence modeling resource webpage [35]. The Reynolds number is 6.0 million and the Mach number is 0.15 . The NASA Langley webpage provides several C-type meshes for performing this simulation. However, since the strand solver cannot operate on such meshes, an O-type 


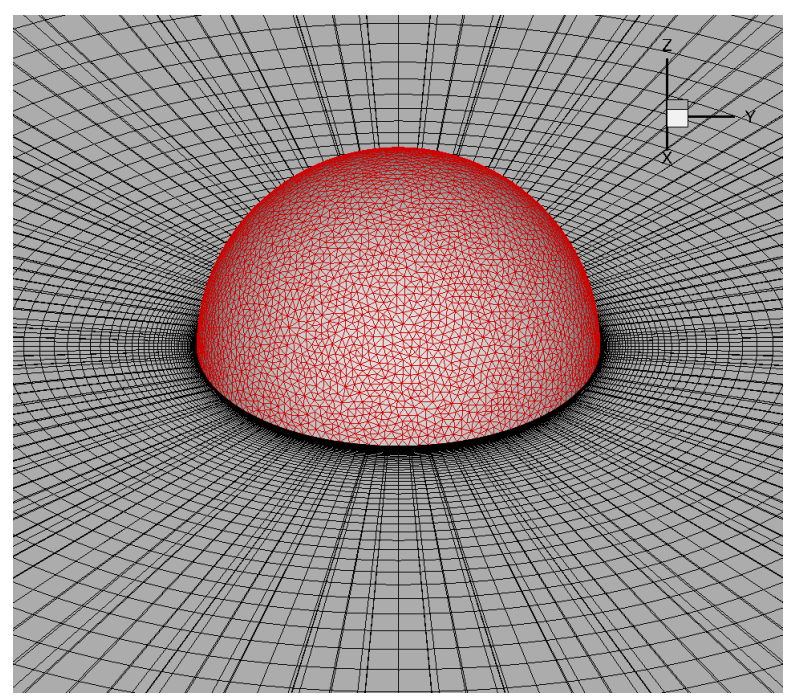

(a) Mesh

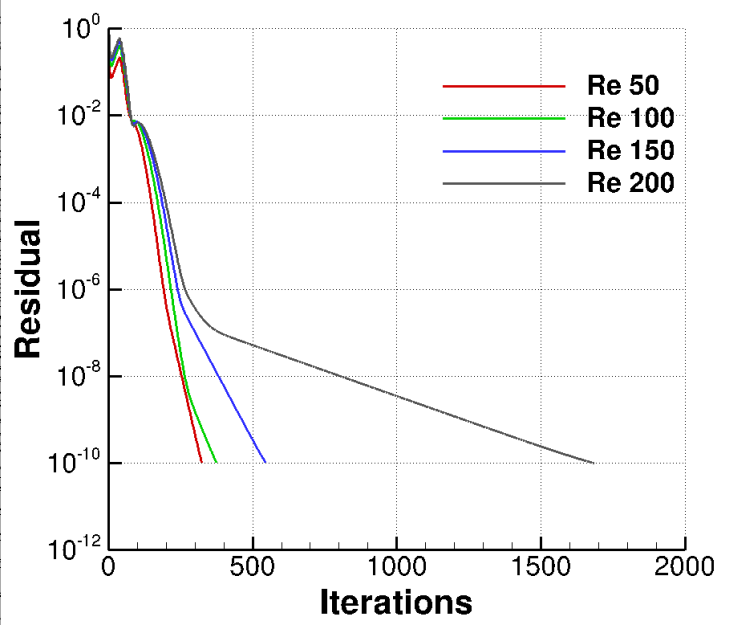

(b) Convergence

Figure 10: Mesh and convergence for flow over a sphere.

mesh generated using a hyperbolic mesh generator with 512 points on the airfoil and 245 points in the strand direction (see Fig. 13(a)). The 2D airfoil mesh is extruded in the third direction to create a 3D mesh with all-quadrilateral surface elements. Symmetry boundary condition is enforced on the sides of the mesh to simulate a quasi-2D flowfield. The mesh extends to a farfield boundary of 500 chords away from the airfoil. The multi-strand solver is again run in isolation. Figure 13(b) shows the convergence of mStrand for three different angles of attack (AoA). In all cases, the code shows good convergence with the residual dropping 8 orders.

Figure 14 shows the comparison of predicted pressure coefficient on the airfoil surface with the experimental data of Ladson [36]. The measurements were obtained by tripping the flow to make it fully turbulent. The CFD and the experimental data show excellent agreement in all cases.

NASA Langley's turbulence resource website also provides the lift and drag coefficients predicted by several flow solvers using SA turbulence model for the considered angles of attack. Table 1 replicates the table with the values obtained using mStrand appended to it. The force coefficients predicted by mStrand is clearly comparable to that predicted by all other flow solvers, thus verifying the implementation of the turbulence model in the strand solver. Note that the strand solver was run on a different grid compared to all other solvers and is expected to have small differences.

\subsection{Transonic Flow Past OneraM6 Wing}

The next test case considered is the transonic flow past OneraM6 wing. The Reynolds number is 11.72 million, Mach number is 0.84 and the angle of attack is $3^{\circ}$. This case is simulated using a mesh consisting of 8977 points on 


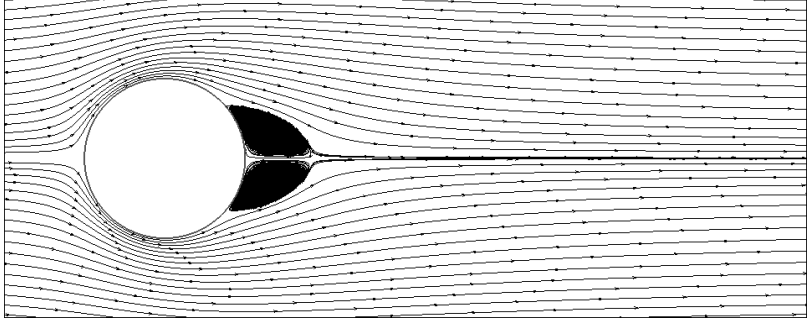

(a) $\mathrm{Re}=50$

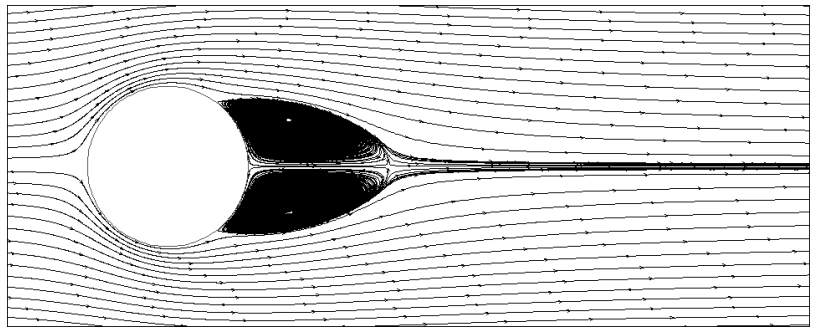

(c) $\operatorname{Re}=100$

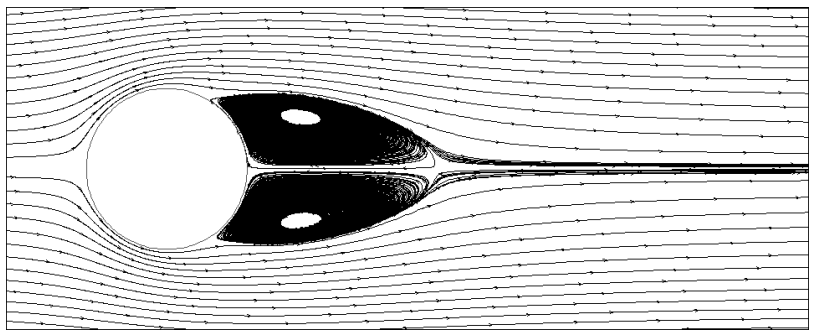

(e) $\operatorname{Re}=150$

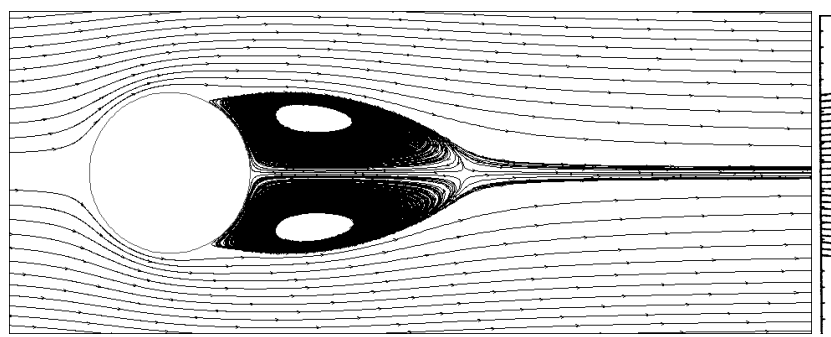

(g) $\operatorname{Re}=200$

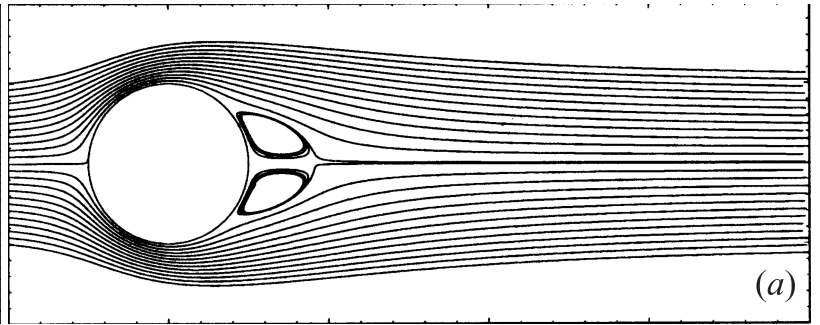

(b) $\mathrm{Re}=50$, Johnson and Patel [31]

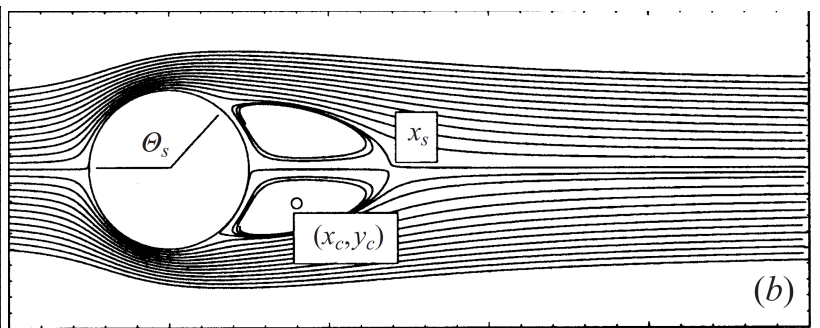

(d) $\mathrm{Re}=100$, Johnson and Patel [31]

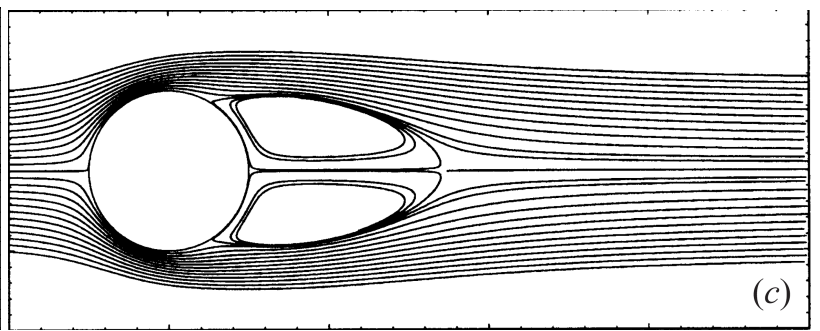

(f) $\mathrm{Re}=150$, Johnson and Patel [31]

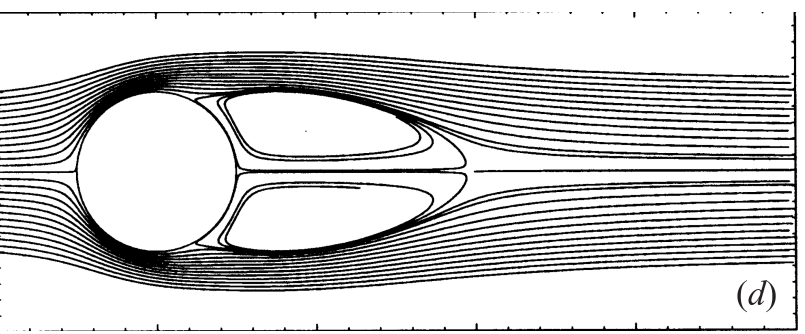

(h) $\mathrm{Re}=200$, Johnson and Patel [31]

Figure 11: Streamlines showing separation bubble for flow over a sphere. 


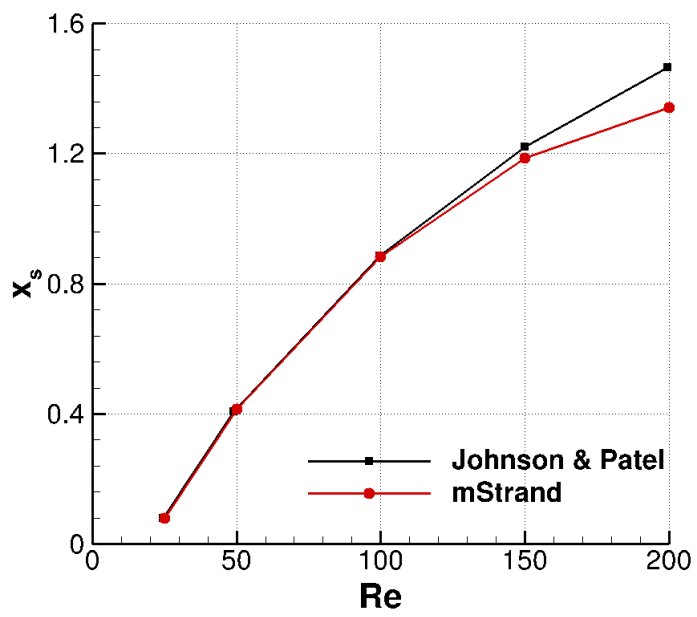

(a) separation distance

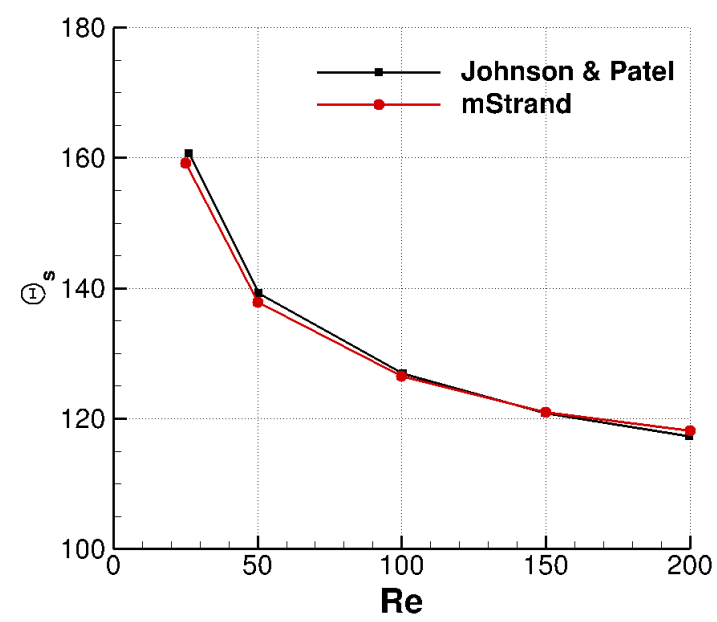

(c) separation angle

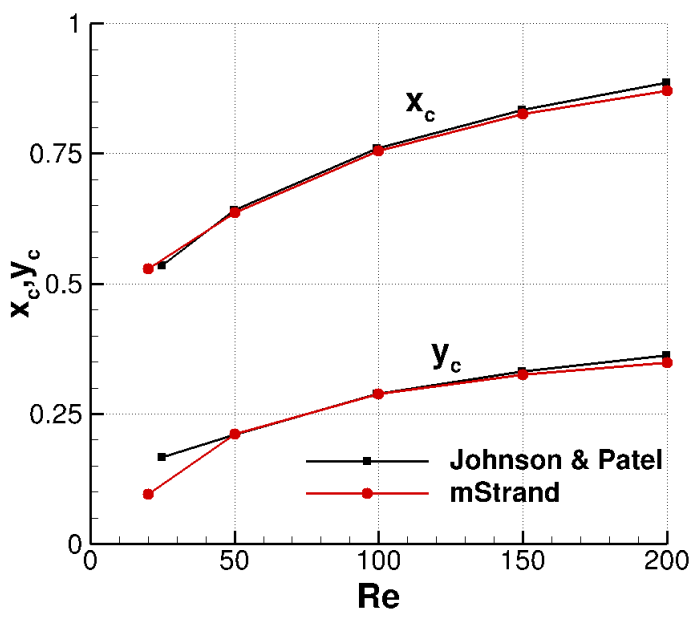

(b) center of separation bubble

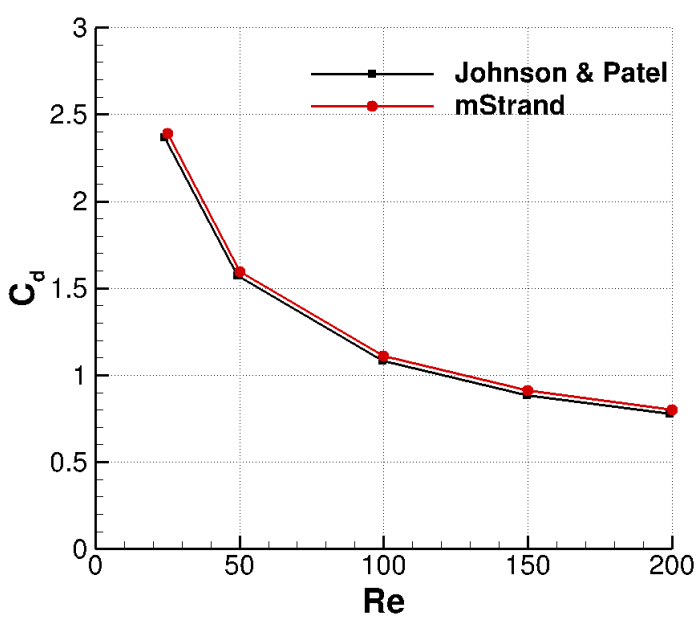

(d) Drag Coefficient

Figure 12: Validation of flow features with the results from Johnson and Patel [31]. 


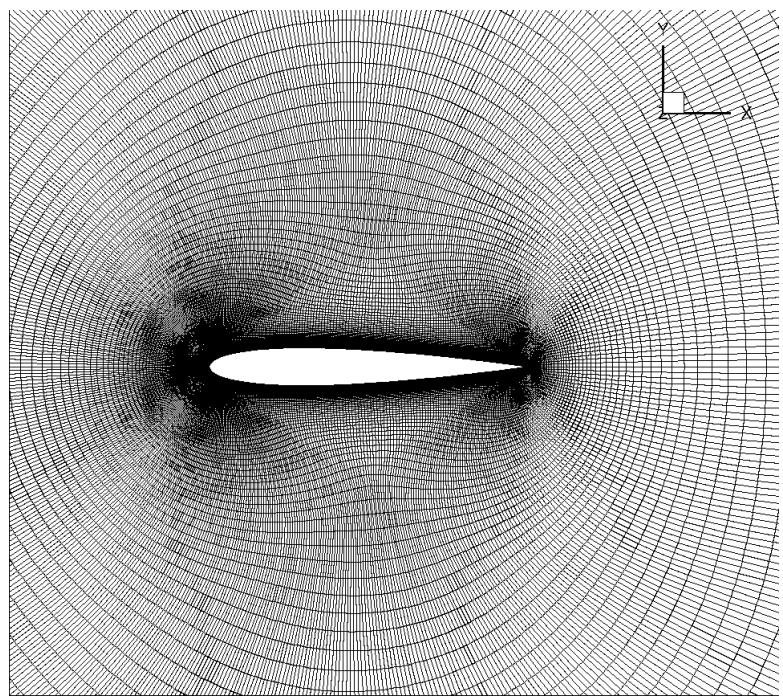

(a) Mesh

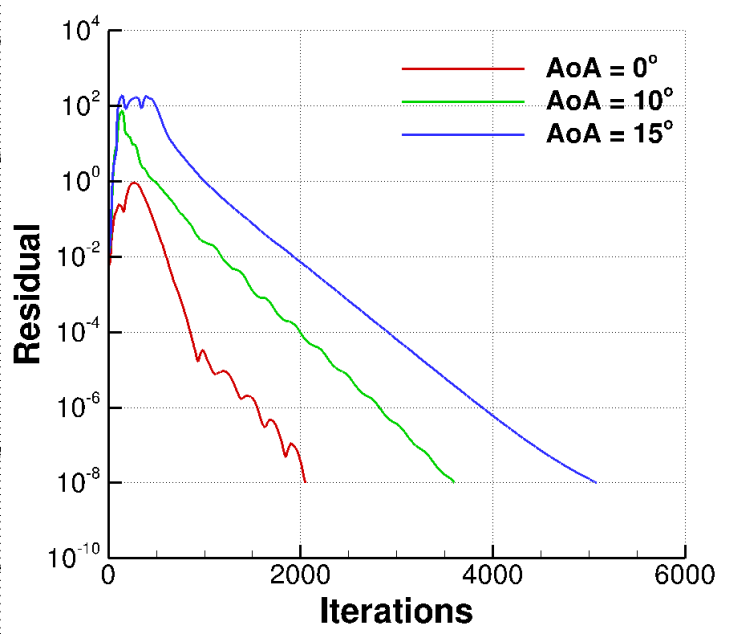

(b) Convergence

Figure 13: Mesh and convergence for NACA0012 airfoil simulation.

\begin{tabular}{|c|c|c|c|c|c|c|}
\hline CODE & $\begin{array}{c}\mathrm{CL} \\
\left(\mathrm{AoA}=0^{\circ}\right)\end{array}$ & $\begin{array}{c}\mathrm{CL} \\
\left(\mathrm{AoA}=10^{\circ}\right)\end{array}$ & $\begin{array}{c}\mathrm{CL} \\
\left(\mathrm{AoA}=15^{\circ}\right)\end{array}$ & $\begin{array}{c}\mathrm{CD} \\
\left(\mathrm{AoA}=0^{\circ}\right)\end{array}$ & $\begin{array}{c}\mathrm{CD} \\
\left(\mathrm{AoA}=10^{\circ}\right)\end{array}$ & $\begin{array}{c}\mathrm{CD} \\
\left(\mathrm{AoA}=5^{\circ}\right)\end{array}$ \\
\hline \hline CFL3D & approx 0 & 1.0909 & 1.5461 & 0.00819 & 0.01231 & 0.02124 \\
\hline FUN3D & approx 0 & 1.0983 & 1.5547 & 0.00812 & 0.01242 & 0.02159 \\
\hline NTS & approx 0 & 1.0891 & 1.5461 & 0.00813 & 0.01243 & 0.02105 \\
\hline JOE & approx 0 & 1.0918 & 1.5490 & 0.00812 & 0.01245 & 0.02148 \\
\hline SUMB & approx 0 & 1.0904 & 1.5446 & 0.00813 & 0.01233 & 0.02141 \\
\hline TURNS & approx 0 & 1.1000 & 1.5642 & 0.00830 & 0.01230 & 0.02140 \\
\hline GGNS & approx 0 & 1.0941 & 1.5576 & 0.00817 & 0.01225 & 0.02073 \\
\hline mStrand & approx 0 & $\mathbf{1 . 0 9 6 7}$ & $\mathbf{1 . 5 6 2 1}$ & $\mathbf{0 . 0 0 8 0 4}$ & $\mathbf{0 . 0 1 2 5 1}$ & $\mathbf{0 . 0 2 1 9 5}$ \\
\hline
\end{tabular}

Table 1: Lift and drag coefficients predicted by different flow solvers for NACA0012 airfoil.

the surface with 3246 triangular elements and 7254 quadrilateral elements. Figure 15(a) shows the surface grid near the tip and the multi-strand mesh near the trailing edge. Two different meshes are generated - 1) extends all the way to the farfield (20 chords away) with 101 points in the strand direction, generated using a multi-level strategy described in Section 4 2) Stops half a chord away with 75 points in the strand direction to transfer the solution to the off-body solver (SAMCART) under Helios framework.

Figure 15(b) shows the convergence of flow solver with the two mesh systems. When mStrand is run in isolation, the residual drops 10 orders in less than 3000 iterations, whereas within the Helios framework it takes 20000 iterations or more. The reason for this is the explicit overset interface between mStrand and the off-body Cartesian solvers, where data exchange happens only at every non-linear iteration. Such characteristics would occur between any two solvers that are explicity coupled and motivates the need for a globally implicit solution option in the future. Nevertheless, 


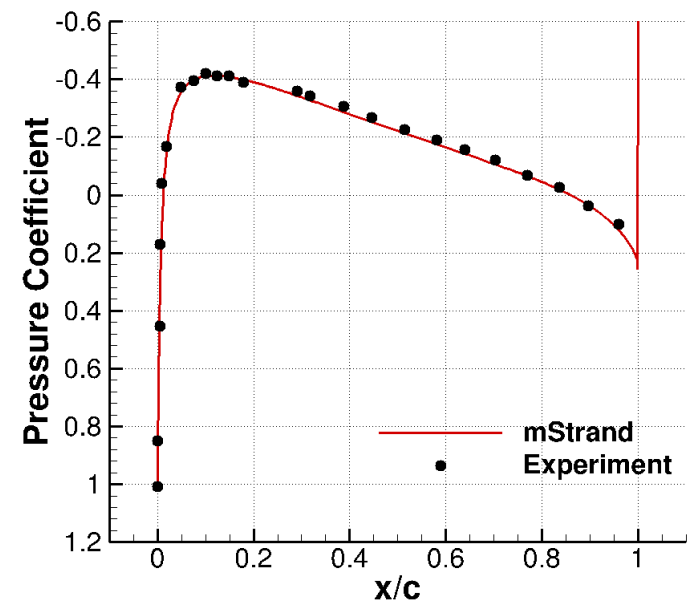

(a) $\mathrm{AoA}=0^{\circ}$

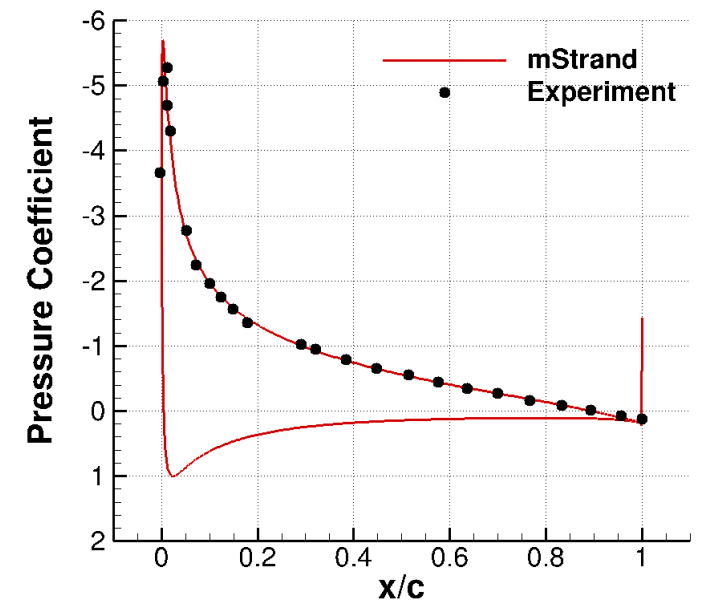

(b) $\mathrm{AoA}=10^{\circ}$

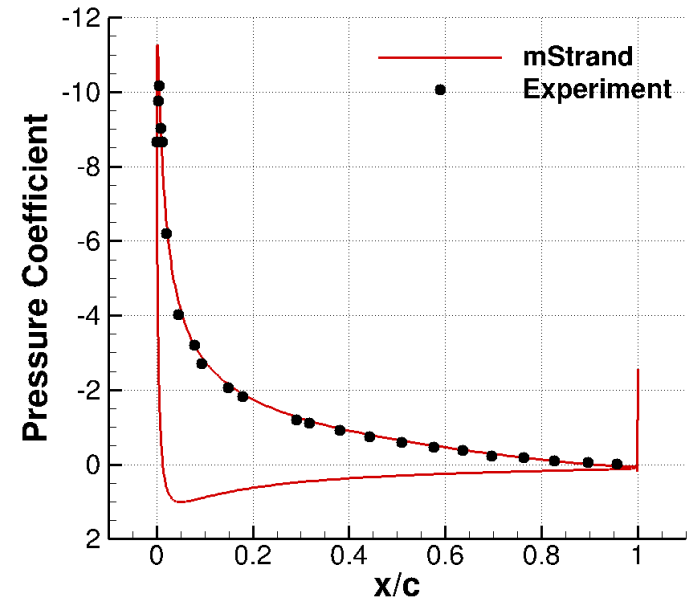

(c) $\mathrm{AoA}=15^{\circ}$

Figure 14: Pressure coefficient comparison for NACA0012 airfoil simulation. 


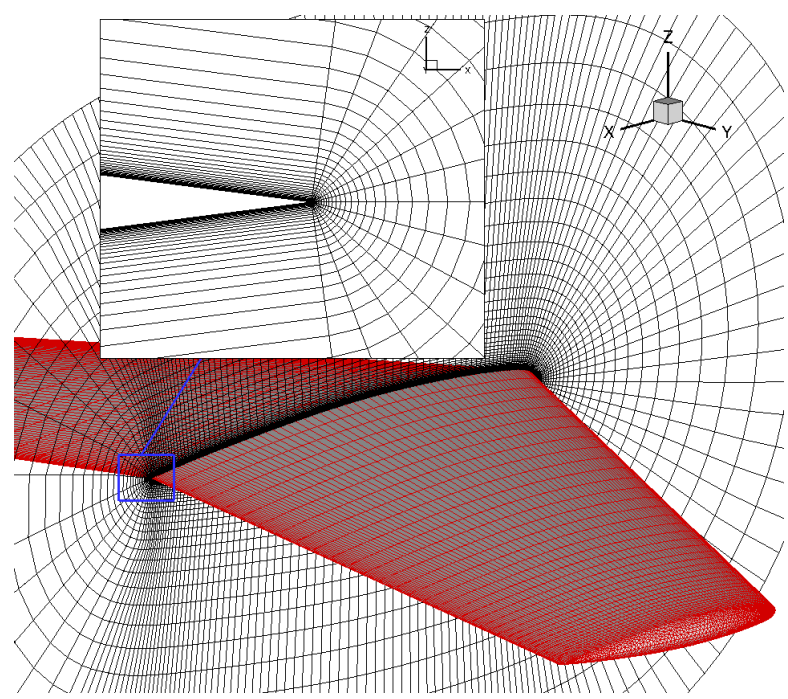

(a) Grid

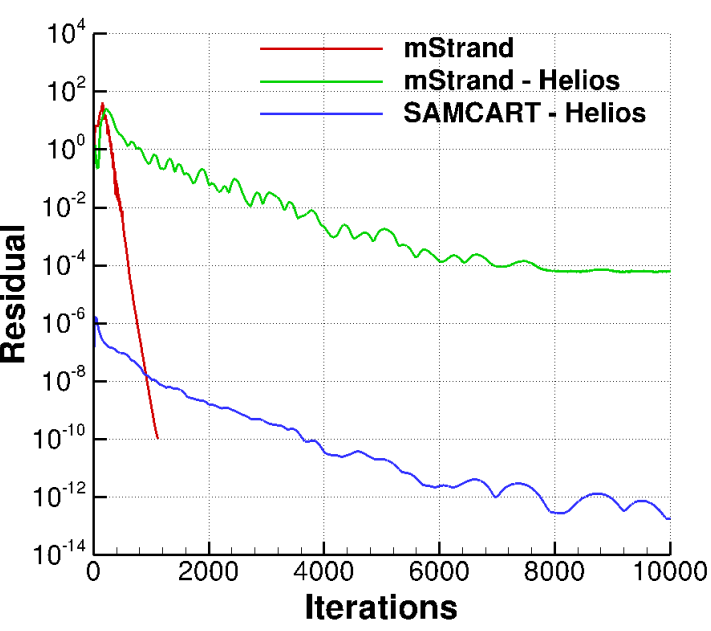

(b) Convergence

Figure 15: Mesh and convergence for OneraM6 wing simulation.

the results reveal that the strand solver is stable on this multi-strand mesh.

Figure 16 shows the sectional pressure contours at different spanwise location as well as the contours across the overset interface. The contours clearly show that the expected lambda-shock is captured. Further, the contours across the near-body and off-body meshes are smooth and continuous, thus verifying the interfacing between mStrand and Helios framework.

A quantitative comparison is provided by plotting the section pressure coefficient with the experimental data provided in Schmitt and Charpin [37] in Fig. 17. Also, plotted are the results from an inviscid simulation. Overall, the results look very satisfactory. The improvement in the prediction with the use of turbulence model is clearly evident. Using a finer mesh might improve the prediction of shock location, but such a study is beyond the scope of this paper. It should be mentioned here that the results obtained with the standalone multi-strand solver and that from Helios framework are almost identical.

\subsection{Validation of Tip Vortex Formation and Evolution in Hover}

To exercise the moving mesh term in mStrand, the next test case considered corresponds to a single-bladed hovering rotor investigated by Martin et al. [38]. The rotor will henceforth be called "Martin rotor" for ease of reference. The tip vortex evolution was studied using LDV-based velocity measurements made at selected wake planes ranging from 3 to $395 \mathrm{deg}$. The setup consisted of single untwisted blade with rectangular planform (with a square tip share) operated on a hover test stand. The relevant test-case details are given in Table 2. Duraisamy et al. [39] provided a 


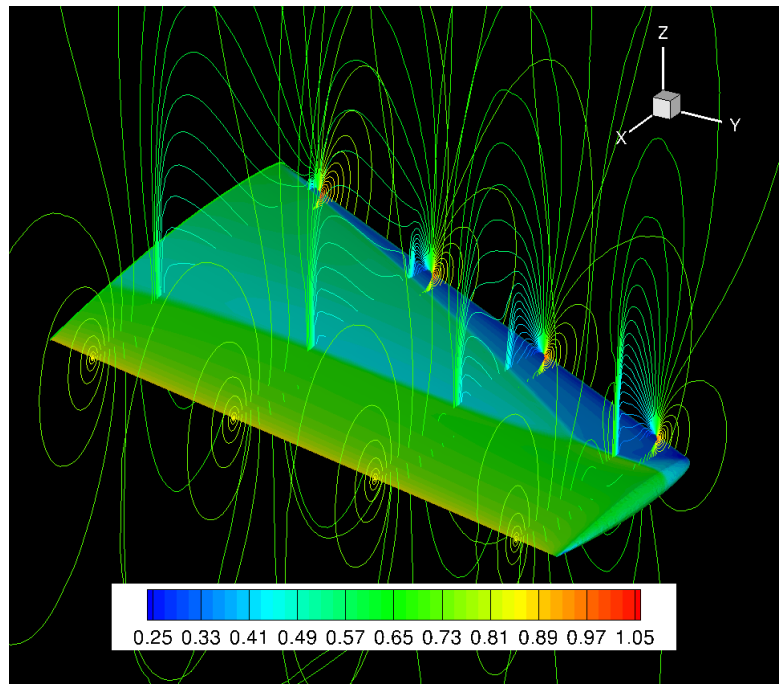

(a) Section pressure contours

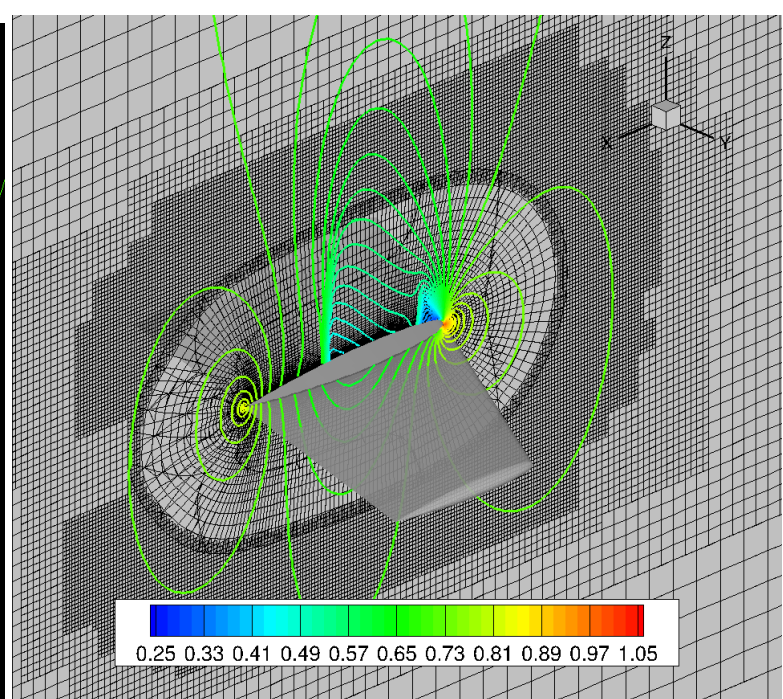

(b) Pressure contour across overset interface

Figure 16: Sectional pressure contour for OneraM6 wing.

\begin{tabular}{|c|c|}
\hline Chord $c$ & $44.5 \mathrm{~mm}$ \\
\hline Blade radius $R$ & $406 \mathrm{~mm}$ \\
\hline Tip Mach Number $M_{\text {tip }}$ & 0.26 \\
\hline Tip Reynolds number $R e_{t i p}$ & 272,000 \\
\hline Collective pitch $\theta$ & $4.5^{\circ}$ \\
\hline Root cutout & $20 \%$ \\
\hline Airfoil & NACA 2415 \\
\hline
\end{tabular}

Table 2: Experimental test conditions for Martin rotor.

detailed study of the tip vortex formation on this exact rotor setup using PIV measurements and correlated the data with their computational study.

The simulation is performed using an off-body Cartesian grid within Helios framework. The near-body grid is a multi-stranded mesh having 34025 grid points on the surface, with 13374 triangular elements and 27336 quadrilateral elements. 81 layers are used in the strand direction. Figure 18 shows the grid near the tip region and the multistrand mesh near the trailing edge. The surface mesh used has quadrilateral elements throughout the blade span and triangular elements near the tip, where generating a quadrilateral mesh is challenging. The strand mesh extends more than quarter-chord in distance, beyond which the solution is transferred to the off-body Cartesian mesh.

The off-body mesh has a resolution of 5\% of the chord length. Calculations are run for 10 revs, 14400 steps with a rotor step of $\Delta \psi=0.25^{\circ}$. The near-body solver uses 8 sub-iterations while the off-body uses 40 sub-iterations. Off-body AMR is turned on after two revs and adapted every $2.5^{\circ}$, or 10 time steps. Convergence of thrust $\left(C_{T}\right)$ 


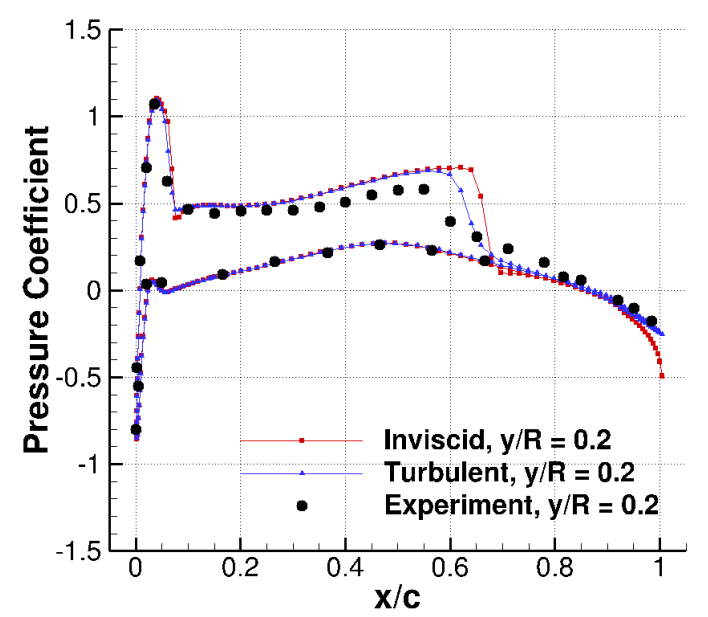

(a) $y / R=0.2$

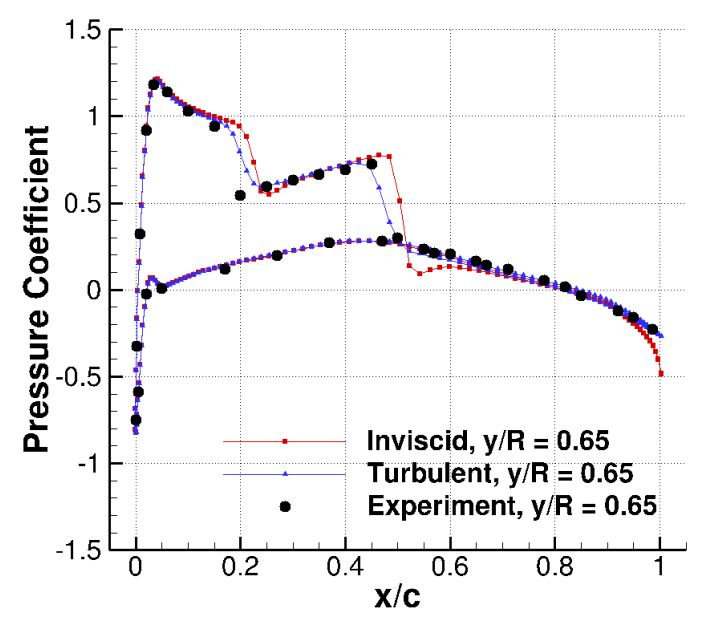

(c) $y / R=0.65$

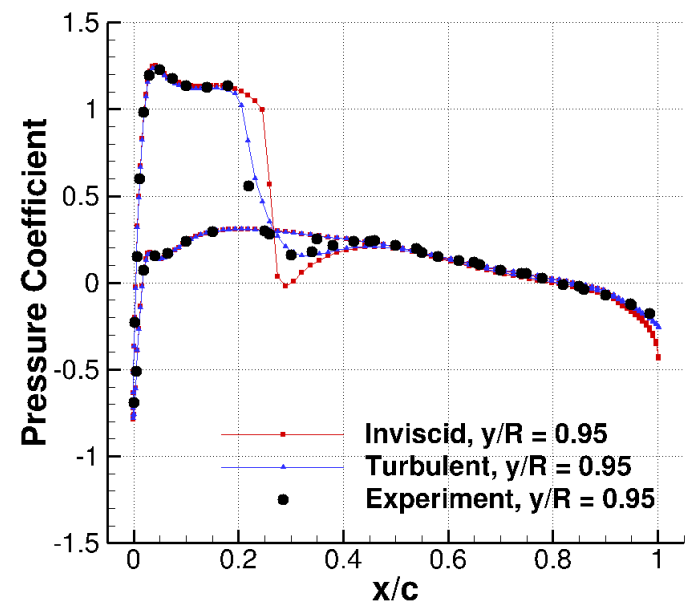

(e) $y / R=0.95$

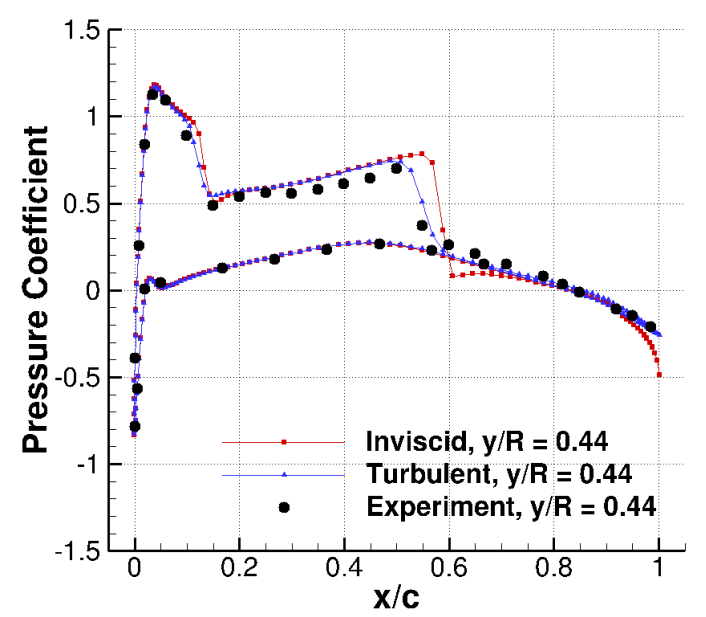

(b) $y / R=0.44$

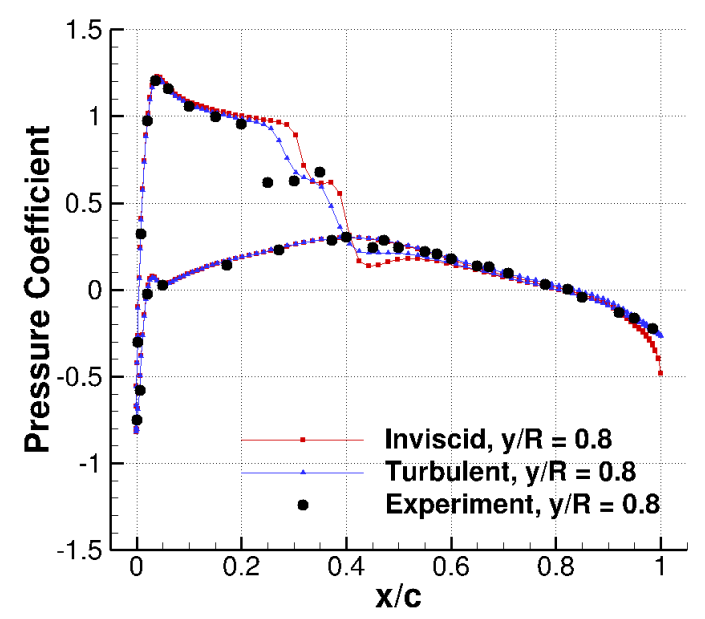

(d) $y / R=0.8$

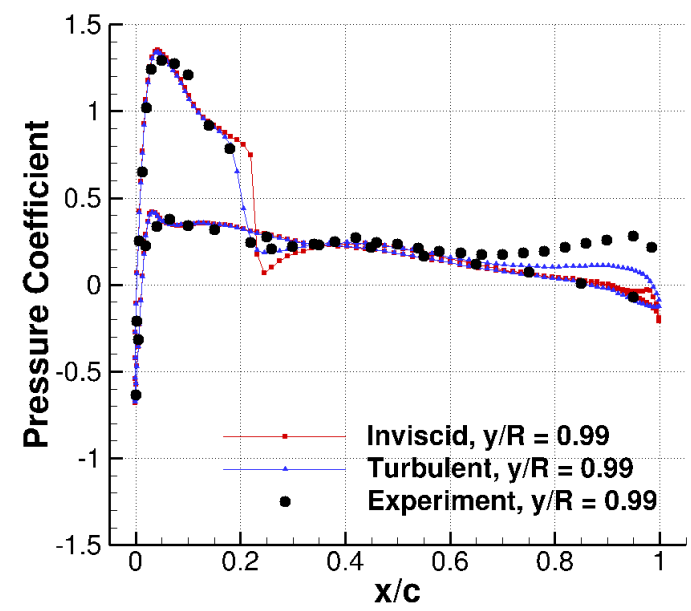

30

(f) $y / R=0.99$

Figure 17: Comparison of sectional pressure coefficient at different spanwise location for OneraM6 wing with the results from Schmitt and Charpin [37]. 


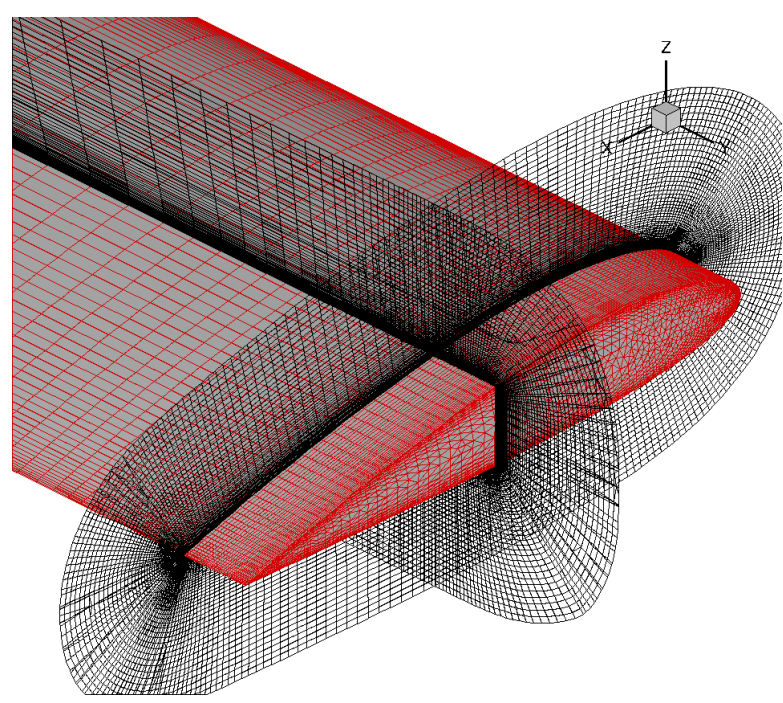

(a) Grid near tip region

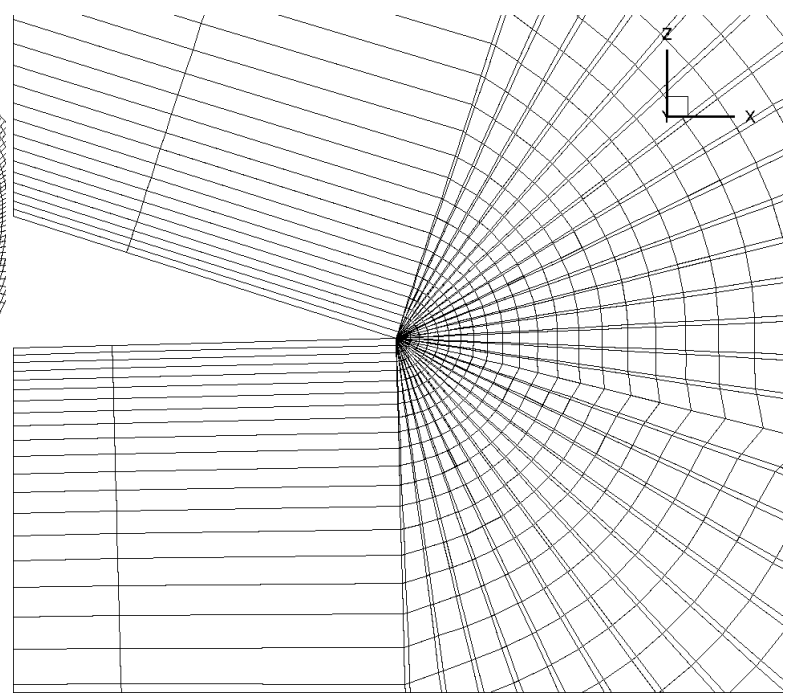

(b) Multi-Stranded at convex corners

Figure 18: Multi-strand mesh for Martin rotor simulation.

and power $\left(C_{Q}\right)$ coefficients is shown in Fig. 19. The rotor thrust coefficient converges close to the experimentally estimated value of 0.002 , giving initial confidence in the simulation.

Figure 20(a) shows the streamwise vorticity contour near the tip of the blade at selected sections. The formation of tip vortex is very well resolved and the presence of secondary and tertiary vortices is evident. Similar conclusion can be drawn from Fig. 20(b), which shows the iso-surface of Q-criterion colored by vorticity magnitude contours. The vortex is transferred smoothly from one mesh to the other. Also seen in this plot is the returning vortex after one blade passage. The twisting of the returning vortex is prominent. The off-body Cartesian mesh has refined adaptively to capture the wake.

Figure 21 provides a quantitative comparison of the captured wake with the experimentally measured swirl velocity (normalized by rotational speed) profile at different wake planes. The swirl velocity profile is obtained by cutting a horizontal line across the center of the vortex in that plane. The $\mathrm{x}$-axis in these plots is the radial location of the vortex normalized by the blade radius. Therefore, the contraction of the wake with increasing wake age is reflected in these plots. The predicted contraction rate is accurate when compared to the experimentally measured value. Examining the vortex profiles, the peak swirl velocity is slightly under-predicted at the earliest wake-age $\left(\xi=3^{\circ}\right)$, which probably results in a slightly larger vortex core. At this wake age, any discrepancy can be primarily attributed to the difficulty in accurately predicting the tip vortex formation using a RANS-based solver. Computational solutions of the RANS equations suffer from numerical and turbulence modeling errors, that can make the prediction of tip vortex formation extremely challenging. It should be noted that these predictions are as accurate as or better than most results available 


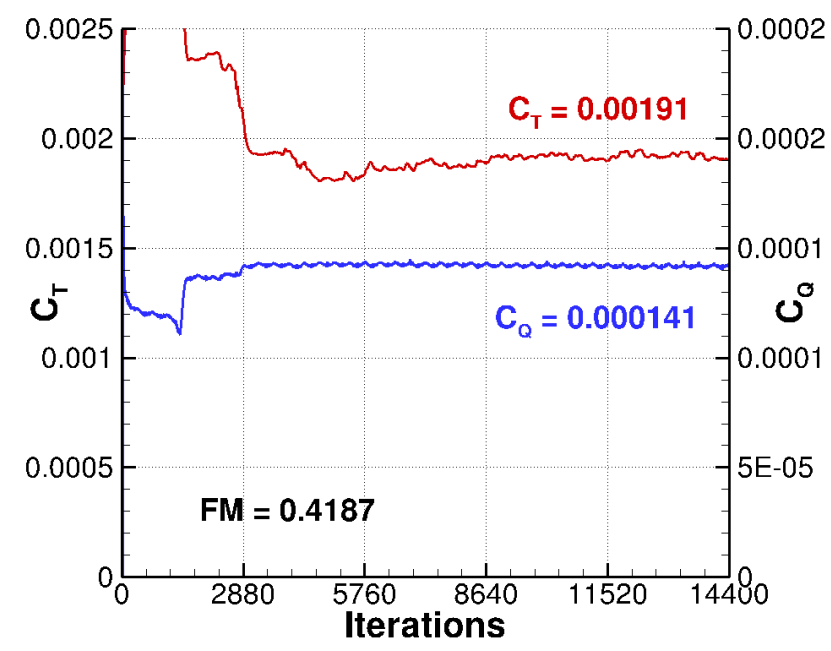

Figure 19: Convergence of thrust and power coefficients for Martin rotor simulation.

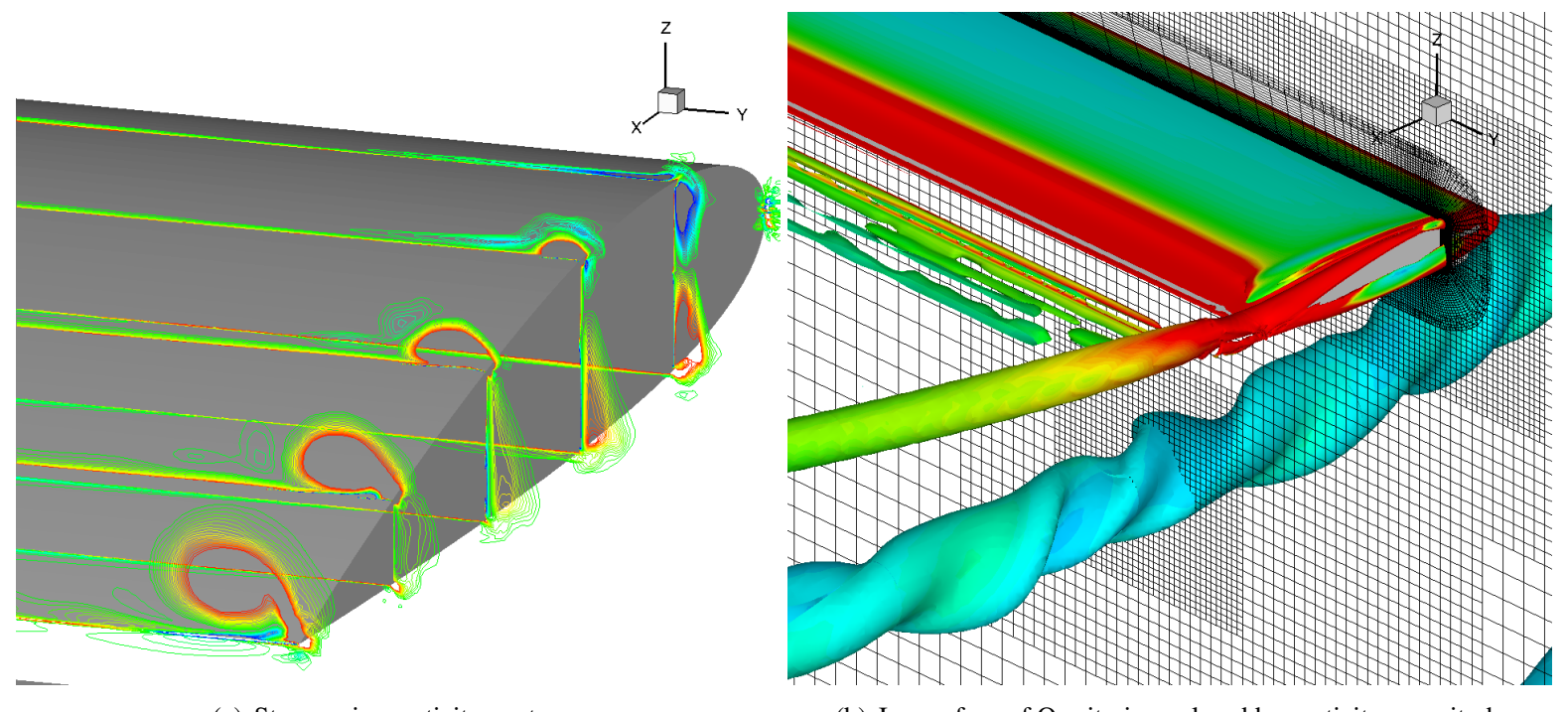

(a) Streamwise vorticity contours

(b) Iso-surface of Q-criterion colored by vorticity magnitude

Figure 20: Formation of trailed vortex system in Martin rotor simulation. 
in the literature.

At other wake ages shown, the tip vortex is resolved in the off-body Cartesian mesh. The high-order Cartesian solver is able to preserve the vortex accurately and the discrepancy between the measurement and the prediction is seen to reduce as the wake-age increases. Note that due to the twisting of the tip vortex (see Fig. 20), the velocity profiles obtained along cuts in different axes are not identical. Some discrepancies in the results could be attributed to that. Overall, the results obtained are very satisfactory and demonstrate that use of a strand-based near body mesh with an off-body Cartesian mesh is a viable framework for accurate prediction of tip vortex formation and evolution.

\subsection{TRAM Rotor in Hover}

Next, the strand solver is evaluated for hover calculations of the isolated Tilt Rotor Aeroacoustics Model (TRAM) rotor, which is a quarter-scale model of the right-hand Bell/Boeing V-22 three-bladed proprotor tested in the DuitsNederlandse Windtunnel Large Low-speed Facility (DNW-LLF). Computed results are compared to the hover tests conducted in airplane mode at tip Mach number 0.62. Aerodynamic surface pressures, thrust and power, were measured along with structural loads and aeroacoustics data. A summary of the nominal operating conditions used for the computational model are shown in Table 3. Further details of the test are given in a comprehensive report by Johnson [40].

The simulation is again performed within the Helios framework. Due to a fine surface tessellation, a single stranded mesh was sufficient to provide the required coverage in the near-body region. All three rotors and the hub are meshed independently. The PUNDIT domain connectivity facilitates parallel data exchange between different near-body meshes. Table 4 provides the statistics of the strand mesh used for the simulation. Similar to the Martin rotor, the blade mesh is comprised mainly of quadrilateral elements with triangular elements only at the tip cap, see Fig. 22(a). The hub mesh is comprised only of triangular elements. The use of quadrilateral and triangular elements in appropriate regions provides a balance between flow solution accuracy and ease of meshing. Figure 22(b) shows interface between a blade, hub and Cartesian mesh. The strand mesh extends to about half the tip chord distance from the blade.

The off-body mesh has a resolution of 5\% of the chord length. Calculations are run for 8 revs, 11520 steps with a rotor step of $\Delta \psi=0.25^{\circ}$. The near-body solver uses 5 sub-iterations while the off-body uses 40 sub-iterations. Off-

\begin{tabular}{|c|c|}
\hline Tip Chord $c$ & 5.5 in \\
\hline Blade Radius $R$ & 57 in \\
\hline Tip Mach Number $M_{t i p}$ & 0.62 \\
\hline Re Number at tip $R e_{t i p}$ & $2.1 \times 10^{6}$ \\
\hline
\end{tabular}

Table 3: Nominal operating conditions for isolated TRAM rotor studies. 


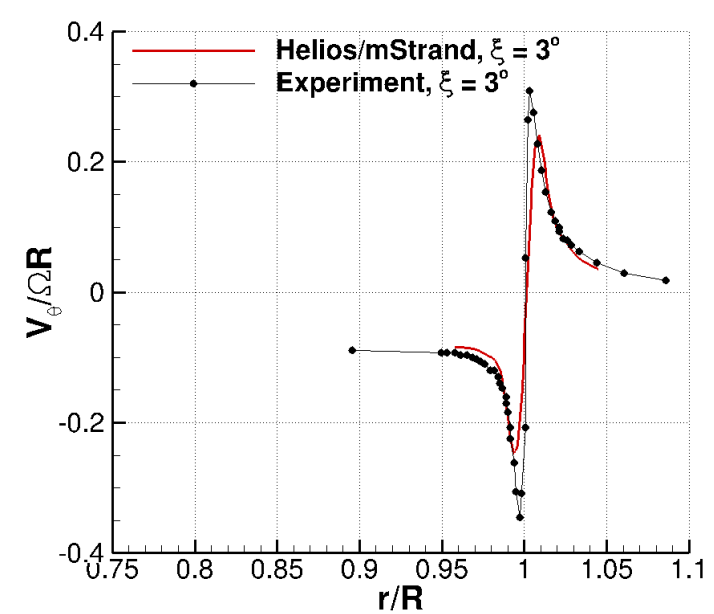

(a) $\xi=3^{\circ}$

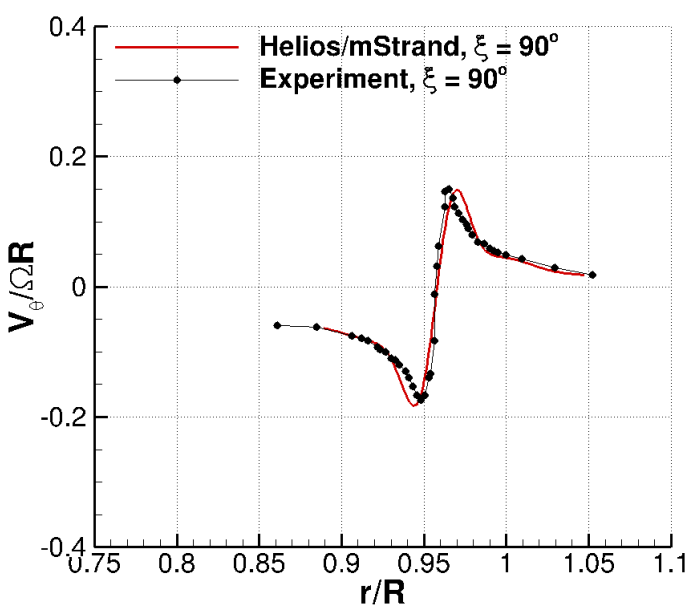

(c) $\xi=90^{\circ}$

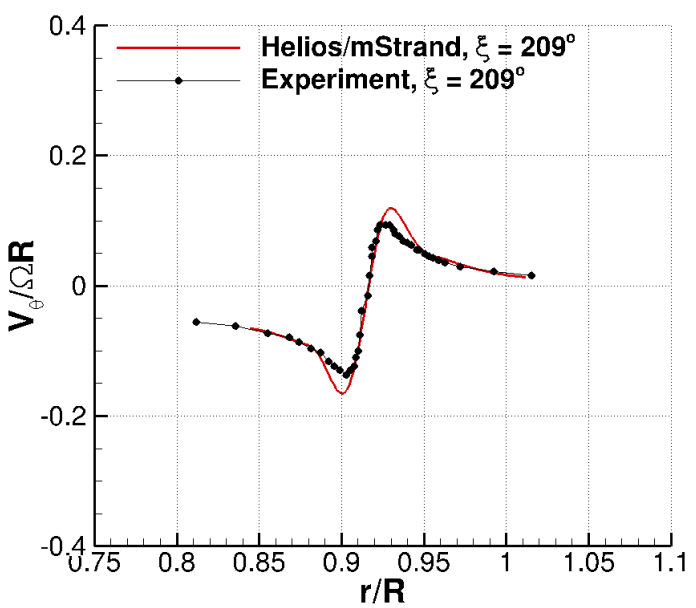

(e) $\xi=209^{\circ}$

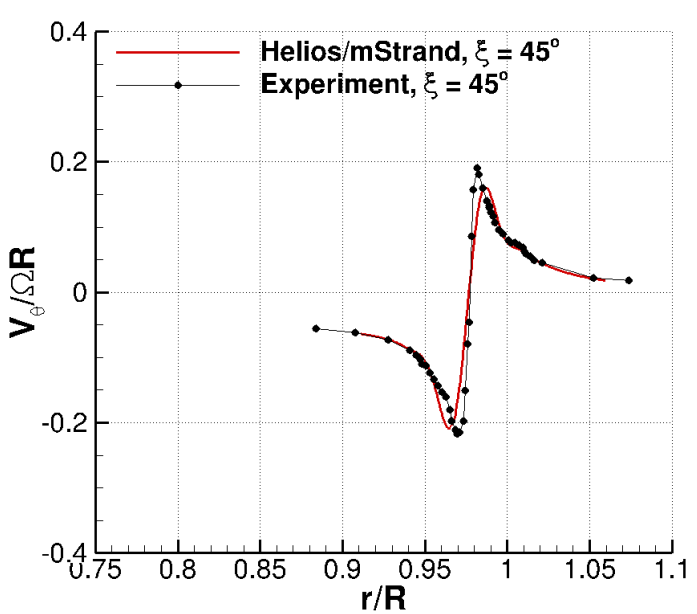

(b) $\xi=45^{\circ}$

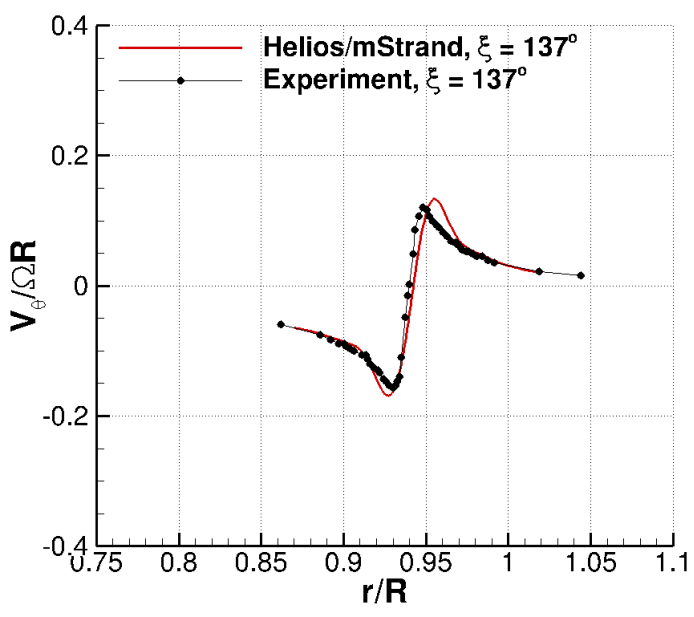

(d) $\xi=137^{\circ}$

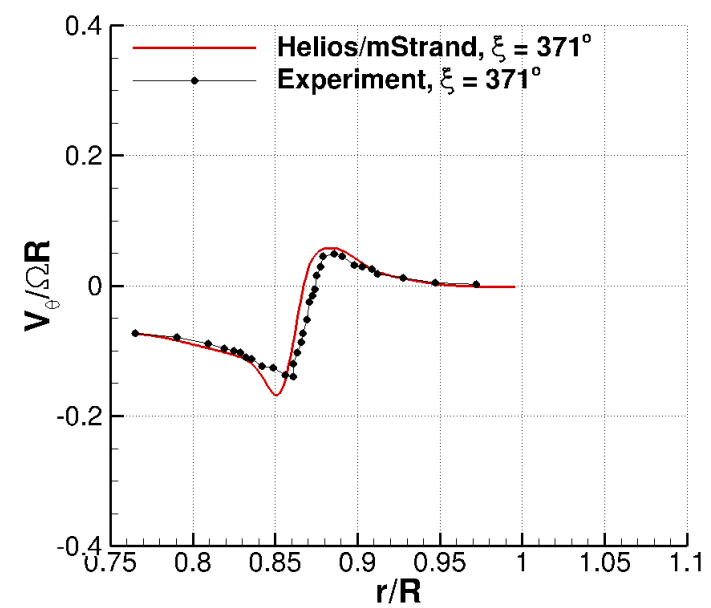

34

(f) $\xi=371^{\circ}$

Figure 21: Swirl Velocity comparison for Martin rotor simulation. 


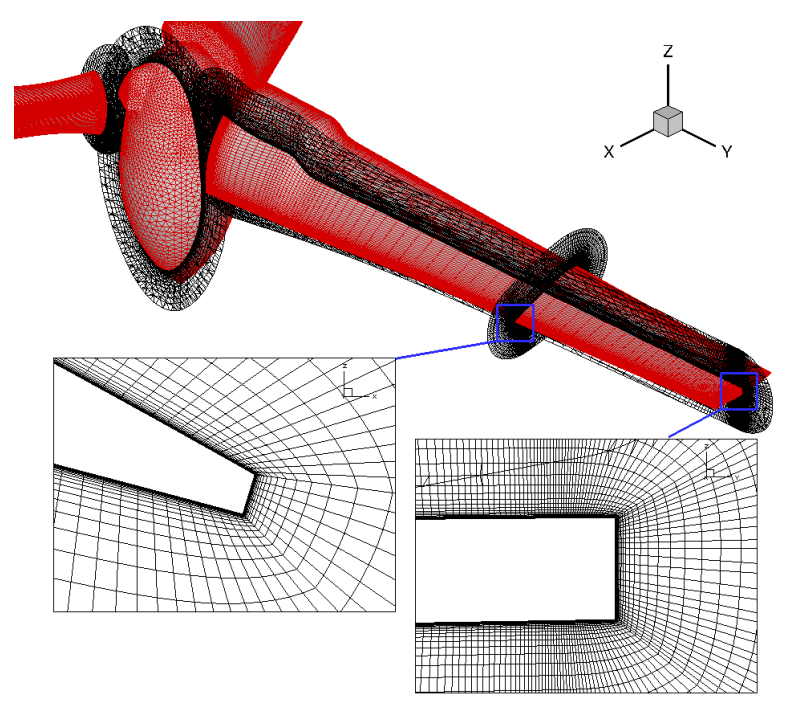

(a) Blade mesh

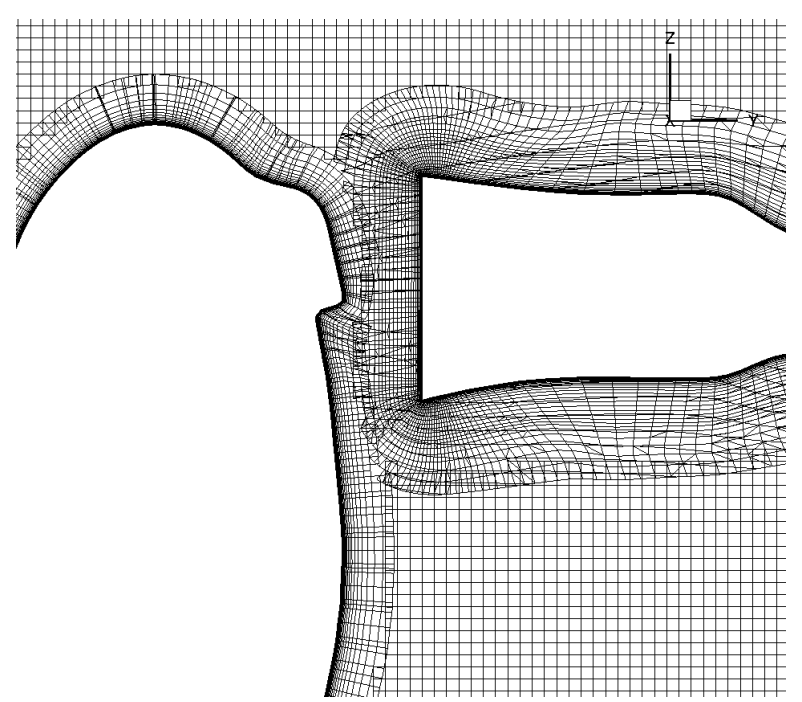

(b) Interface between blade, hub and Cartesian mesh

Figure 22: Mesh for TRAM rotor simulation.

body AMR is turned on after two revs and adapted every $2.5^{\circ}$, or 10 time steps. Figure. 23 shows the figure of merit ( $F M$, a measure of rotor efficiency) and sub-iteration convergence of the solver for the simulation at $14^{\circ}$ collective angle. The forces converge fairly well in 5-6 revs and the strand solver residual converges two-orders of magnitude every time-step.

Figure 24 show the variation of power coefficient $\left(C_{Q}\right)$ with thrust coefficient $\left(C_{T}\right)$ and the variation of figure of merit $\left(F M=\frac{1}{\sqrt{2}} \frac{C_{T}^{\frac{3}{2}}}{C_{Q}}\right)$ with $C_{T}$, obtained by changing the collective angle of the blade. Plotted are the results from the experiments, Helios/mStrand setup and the results obtained using an unstructured near-body flow solver (NSU3D [41]) in the Helios framework [42]. The Helios/NSU3D calculation is the best quality simulation performed so far for this rotor with the Helios framework. The results clearly show that the performance predicted by the strand solver is comparable to the benchmark best available predictions. Both the CFD calculations under-predict the $F M$ by $2-3 \%$ at intermediate collective angles. Overall, the results from the strand solver in Helios framework are very satisfactory for this rotor.

Figure 25 shows visualizations of the computed wake for the $\theta=6^{\circ}, \theta=10^{\circ}$ and $\theta=14^{\circ}$ collective conditions.

\begin{tabular}{|l|c|c|c|c|}
\hline Mesh & $\begin{array}{c}\text { Surface } \\
\text { Nodes }\end{array}$ & $\begin{array}{c}\text { Triangular } \\
\text { Elements }\end{array}$ & $\begin{array}{c}\text { Quadrilateral } \\
\text { Elements }\end{array}$ & $\begin{array}{c}\text { Strand } \\
\text { Points }\end{array}$ \\
\hline blade & 42004 & 6824 & 38590 & 51 \\
hub & 4495 & 8986 & 0 & 51 \\
\hline
\end{tabular}

Table 4: TRAM mesh statistics. 


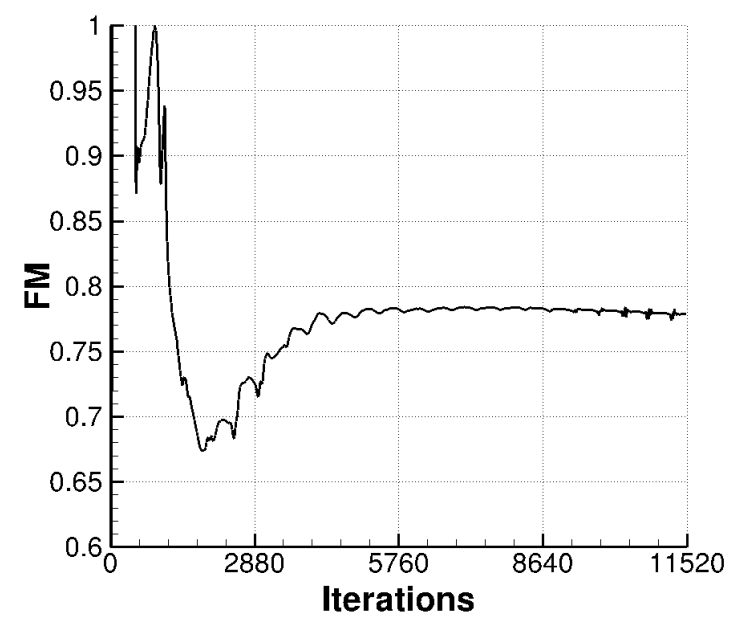

(a) Figure of merit convergence

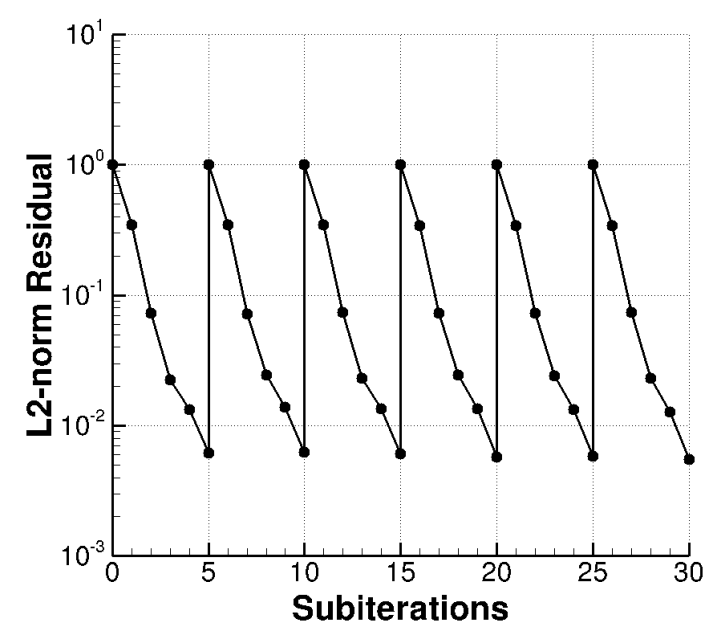

(b) Sub-iteration convergence

Figure 23: Figure of merit and sub-iteration convergence for TRAM rotor simulation.

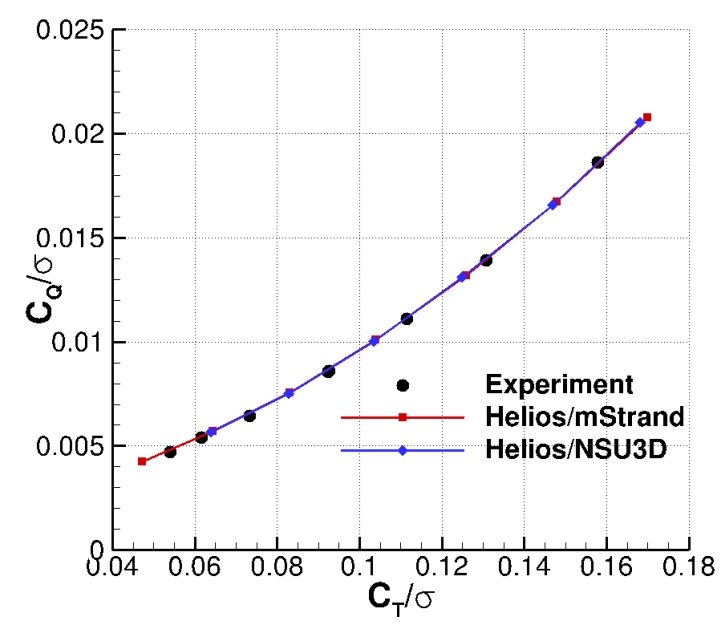

(a)

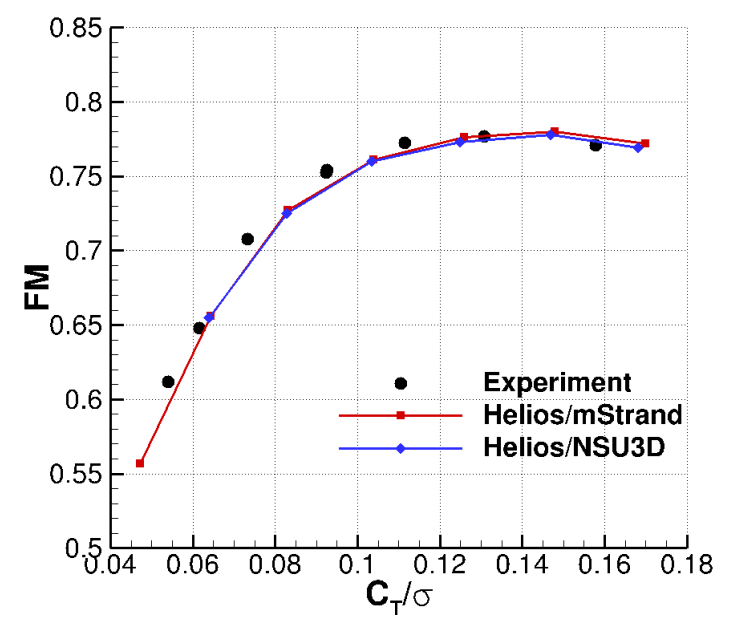

(b)

Figure 24: Performance results from TRAM rotor simulation. 
The wake is very well predicted in all cases. The $\theta=6^{\circ}$ collective case has a tightly confined wake with the tip vortex from the preceding blade directly impacting near the tip. At higher collective angles, the wake gets more spread out and the vortex passes well underneath the blade. The flow visualization shows tip vortices from different blade passages interacting with each other as well as the vortices getting twisted similar to those in the Martin rotor case. The results demonstrate the ability of mStrand to handle very complex problems and the maturity of the flow solver to be used in a production-level environment.

\section{Code Performance Study}

In this section, the performance of the mStrand flow solver is assessed. For this purpose, time-dependent simulation of the TRAM rotor, discussed in Section 5.5, is simulated without the hub. The blade is set at $14^{\circ}$ collective angle. The simulation is done on different number of processors ranging from 180 to 1440 . For a comparative study, a similar set of simulations are also performed using two other flow solvers that are part of Helios - 1) NSU3D [41], which is an unstructured flow solver developed at University of Wyoming, 2) OVERFLOW [43], which is a structured grid solver developed at NASA Langley Research Center.

In order to have a fair comparison between different solvers, it is necessary to provide them with identical meshes. The mesh for NSU3D can be obtained by directly converting the strand mesh to the required unstructured format. However, the authors had difficulty getting NSU3D to work with quadrilateral surface elements. As a solution, each quadrilateral element is split into two triangles, which means each hexahedral volume element is split into two prisms. This process does not increase the total number of degrees of freedom, but it does increase the total number of edges. Therefore, it warrants to verify that the use of the triangulated mesh does not have significant effect on the performance of a flow solver. Consequently, mStrand calculations were repeated on this modified mesh and it was found to have only a minimal performance degradation of $\approx 5 \%$ when compared to using the original mixed quad/tri mesh. Thus, the authors believe that it is reasonable to use the triangulated mesh with NSU3D for performance comparison.

For the simulations with OVERFLOW, some modifications to the mesh is needed. Recall that in the strand mesh, quadrilateral surface elements are used in the entire span of the blade. Therefore, it is straightforward to convert the mesh along the span of the blade to a structured O-mesh. However, triangular elements are used at the root and the tip caps. In the OVERFLOW mesh, the triangular elements are removed and replaced by cap meshes. During this process, the total number of mesh points are kept comparable. Note that the use of cap meshes is a standard practice when OVERFLOW is used for simulating rotor blades. All the mesh systems used approximately 6.4 million nodes.

All the flow solvers are simulated for 100 time-steps using different number of processors. Two orders of convergence per time-step are sought after in all flow solvers; at the same, a limit on the maximum number of sub-iteration is 


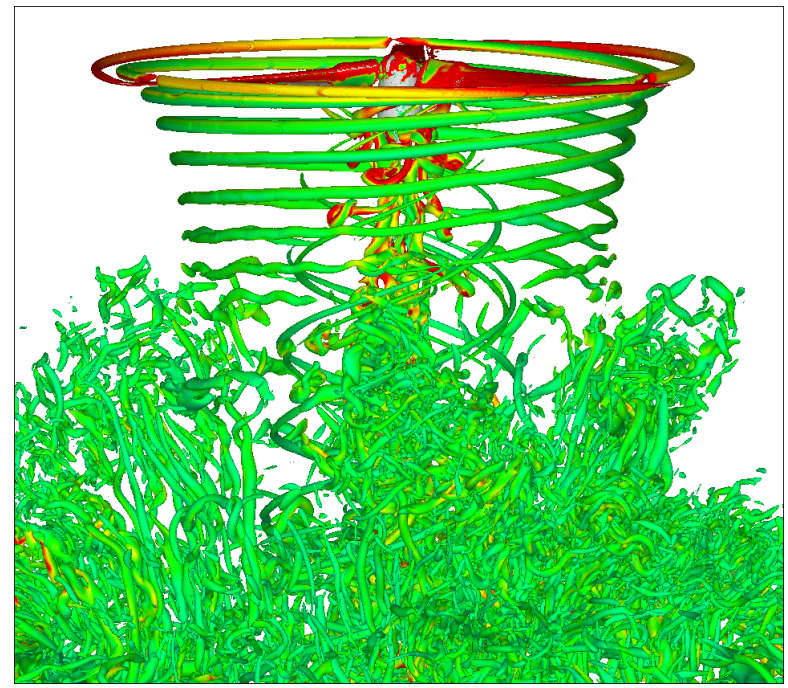

(a) $\theta=6^{\circ}$

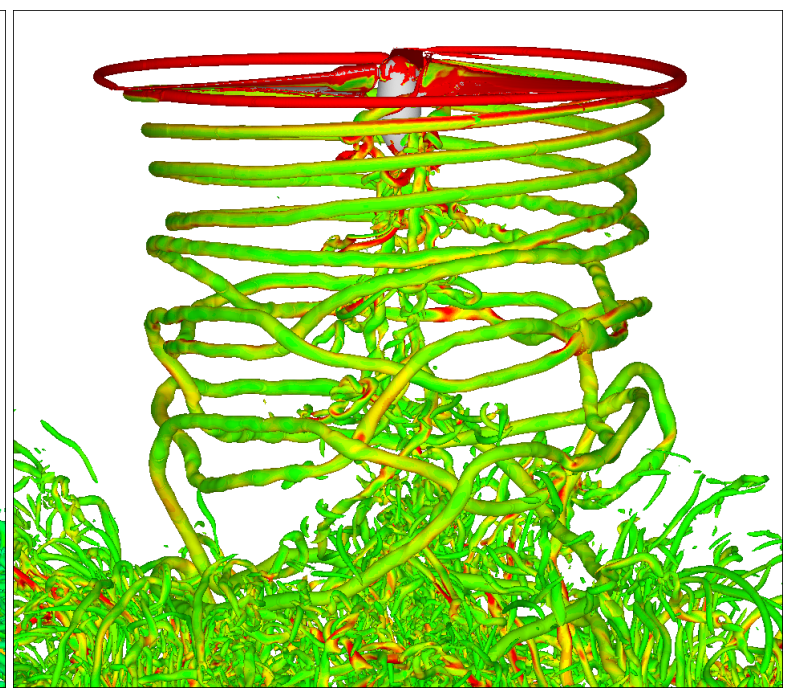

(b) $\theta=10^{\circ}$

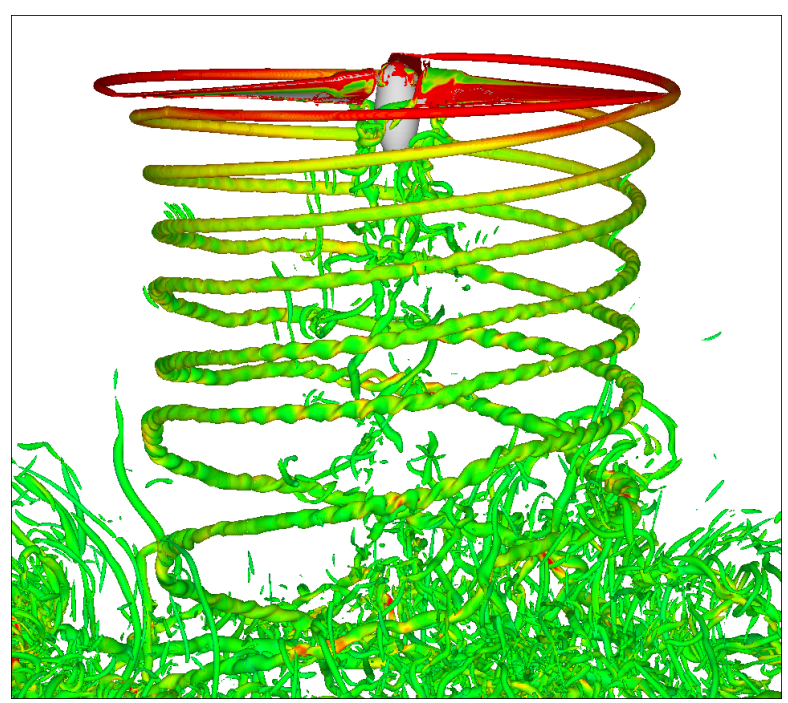

(c) $\theta=14^{\circ}$

Figure 25: Flow visualization using iso-surface of Q-criterion colored by vorticity magnitude for TRAM rotor simulation. 


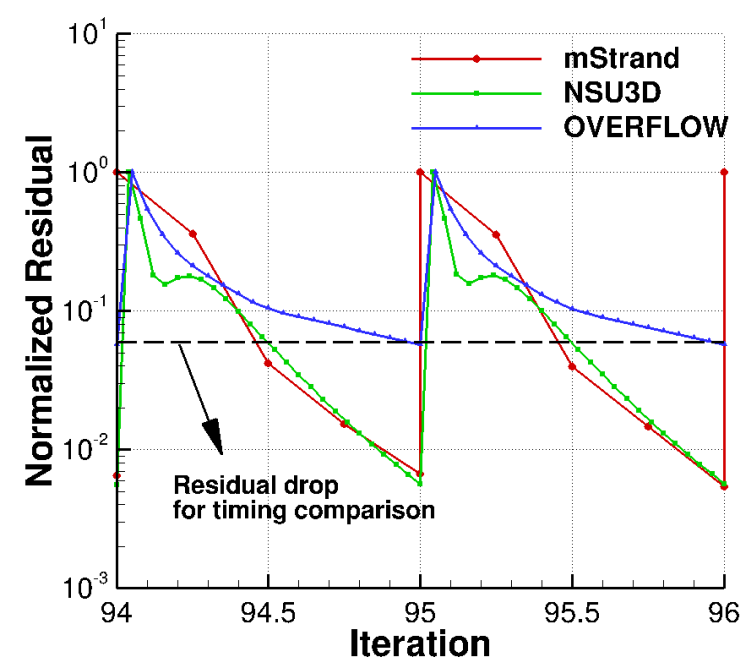

Figure 26: Residual convergence of different solvers for TRAM rotor simulation.

set to prevent prohibitively large computational time. To the best of authors' knowledge, all the flow solvers are set to use their most efficient algorithms. Figure 26 shows the convergence of flow residuals of all the flow solvers for couple of time-steps. The mStrand and NSU3D runs are able to converge to the specified tolerance, but the OVERFLOW run converge to a lower degree in the set maximum number of sub-iterations. For obtaining an unbiased result, the wall-clock time is compared at the same level of convergence (shown with a black dashed line in the figure). This corresponds to 2 sub-iterations in mStrand, 12 sub-iterations in NSU3D and 20 sub-iterations in OVERFLOW. The wall-clock time per time-step is calculated as an average value over the last 10 time-steps simulated.

Figure 27(a) shows the comparison of wall-clock time per time-step for all three solvers. It should be mentioned here that the OVERFLOW wall-clock time only includes the flow solver timing and not the time required for domain connectivity between the blade and the cap meshes, which is performed by OVERFLOW's internal domain connectivity engine. The time for domain connectivity could be significant, especially when using large number of processors, and could skew the results if considered. In Fig. 27(a), both the structured and the strand grid solvers outperforms the unstructured grid solver at all processor counts. On the other hand, while OVERFLOW outperforms strand solver when run on smaller number of processors, the strand solver performs the best as the processor count increases. The reasoning for this can be seen from Fig. 27(b). Like NSU3D, mStrand achieves close to 100\% parallel efficiency, whereas OVERFLOW achieves much less speed up and therefore, is less efficient.

The above results are from a single test case, not a comprehensive study and further studies are needed to make any solid conclusion. But, these initial results do highlight some of the advantages of a strand-based solver. A strand 


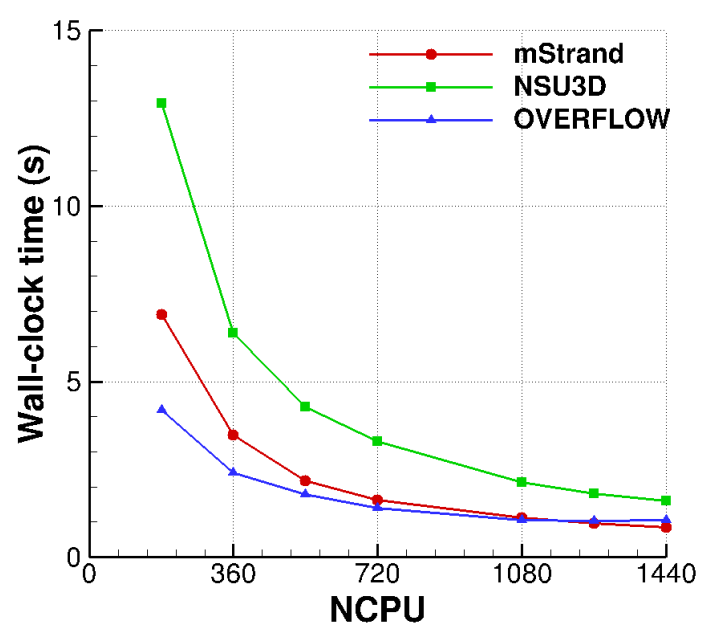

(a) Timing comparison

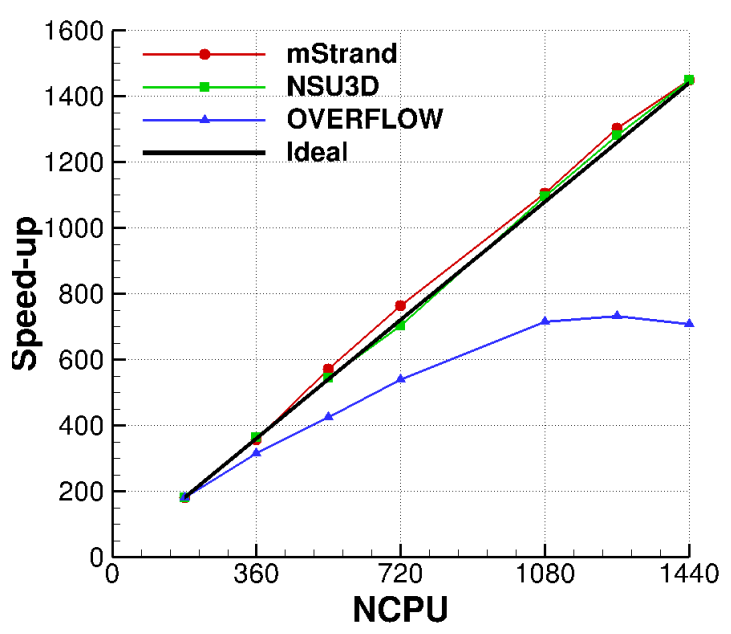

(b) Parallel scaling comparison

Figure 27: Comparison of performance of different solvers for TRAM rotor simulation.

based solver is slower than a structured mesh solver, especially at smaller processor counts, but generating strand mesh for complex geometries is significantly easier than its structured counterpart. It provides preliminary evidence that the strand solver can scale comparably to an unstructured solver to large number of cores and can provide an overall faster turn-around time.

\section{Concluding Remarks}

In this paper, a fully parallel, highly efficient, robust multi-strand solver (mStrand) is introduced. The methodology used in the flow solver is provided in detail. A simple and elegant approach of robustly handling multiple strands, without altering the data structure is presented, where the surface nodes with multiple strands are replicated. Accuracy analysis of the solver using Method of Manufactured solution yielded second-order accuracy for both surface and strand refinements.

The multi-strand solver is integrated with HPCMP CREATE ${ }^{\mathrm{TM}}$-AV Helios framework to simulate complex aerodynamic flows. Several test cases including laminar flow past sphere, turbulent flow past NACA0012 airfoil, transonic flow past OneraM6 wing, tip vortex formation and evolution of a single-bladed hovering rotor and 3-bladed hovering TRAM rotor are simulated. The computed results match experimental data to the same degree of accuracy as other production solvers in Helios such as NSU3D and OVERFLOW. The results from these cases demonstrated the accuracy and stability of the flow solver.

The performance and parallel scalability of the flow solver was tested by performing TRAM rotor simulation on 
different number of processors and comparing the results with established unstructured and structured grid solvers. The results indicate that the strand solver is faster than an unstructured solver but scales comparably. The structured solver does outperform the strand solver at low processor count. But because of its better scalability, the strand solver performs the best at large processor counts.

As a closing remark, the authors believe that the strand technology is promising. The strand mesh generation process can be automated to the most part, similar to that for unstructured grids; at the same time strand solvers can be more efficient than unstructured solvers and can possibly outperform even the structured grid solvers to provide the best turnaround time.

\section{Acknowledgments}

Material presented in this paper is a product of the CREATE-AV Element of the Computational Research and Engineering for Acquisition Tools and Environments (CREATE) Program sponsored by the U.S. Department of Defense HPC Modernization Program Office. This work was conducted at the High Performance Computing Institute for Advanced Rotorcraft Modeling and Simulation (HIARMS). The authors would like to acknowledge Mr. Mark Potsdam for providing help in generating the grid system used for the OVERFLOW calculations.

\section{References}

[1] Meakin, R., Wissink, A., Chan, W., Pandya, S., and Sitaraman, J., "On Strand Grids for Complex Flows,” AIAA-2007-3834, 18th AIAA Computational Fluid Dynamics Conference, Miami, FL, June 2007.

[2] Katz, A., Wissink, A., Sankaran, V., Meakin, R., and Chan, W. "Application of Strand Meshes to Complex Aerodynamic Flowfields," Journal of Computational Physics, Vol. 230, No. 17, 2011.

[3] Sitaraman, J., Roget, B., and Wissink, A., "OSCAR - An Overset Grid Assembler for Overlapping Strand/Cartesian Mesh Systems," 11th Symposium on Overset Composite Grids and Solution Technology, Dayton, OH, October 2012.

[4] Wissink, A., Katz, A., Chan, W., and Meakin, R. "Validation of the Strand Grid Approach," AIAA-2009-3792, 19th AIAA Computational Fluid Dynamics Conference, San Antonio, TX, June 2009.

[5] Katz, A., Wissink, A., and Sankaran, V., "Convergence Acceleration Techniques for Coupled Adaptive Cartesian-Strand Grid Solutions," AIAA-2011-772, 49th AIAA Aerospace Sciences Meeting, Orlando, FL, January 2011.

[6] Katz, A., and Wissink, A., "Efficient Solution Methods for Strand Grid Applications," 30th AIAA Applied Aerodynamics Conference, New Orleans LA, June 2012.

[7] Work, D., Tong, O., Katz, A., and Wissink, A., "Strand Grid Solution Procedures for Sharp Corners," AIAA-2013-0800, 51st AIAA Aerospace Sciences Meeting, Grapevine TX, January 2013.

[8] Katz, A., and Work, D., "High-Order Flux Correction/Finite Difference Schemes for Strand Grids," 52nd AIAA Aerospace Sciences Meeting, National Harbor, MD, January 2014.

[9] Haimes, R., "MOSS: Multiple Orthogonal Strand System," Proceeding of the 22nd International Meshing Roundtable, Springer-Verlag, pp. 75-91, 2013. 
[10] Wissink, A., Jayaraman, B., Datta, A., Sitaraman, J., Potsdam, M., Kamkar, S., Mavriplis, D., Yang, Z., Jain, R., Lim, J., and Strawn, R., “Capability Enhancements in Version 3 of the Helios High-Fidelity Rotorcraft Simulation Code," AIAA-2012-0713, 50th AIAA Aerospace Sciences Meeting, Nashville, TN, January 2012.

[11] Sankaran, V., Wissink, A., Datta, A., Sitaraman, J., Jayaraman, B., Potsdam, M., Katz, A., Kamkar, S., Roget, B., Saberi, H., Chen, W., Johnson, W., and Strawn, R., "Overview of the Helios Version 2.0 Computational Platform for Rotorcraft Simulations,” AIAA-2011-1105, 49th AIAA Aerospace Sciences Meeting, Orlando, FL, January 2011.

[12] Sankaran, V., Sitaraman, J., Wissink, A., Datta, A., Jayaraman, B., Potsdam, M., Mavriplis, D., Yang, Z., O’Brien, D., Saberi, H., Cheng, R., Hariharan, N., and Strawn, R., "Application of the Helios Computational Platform to Rotorcraft Flowfields," AIAA-2010-1230, 48th AIAA Aerospace Sciences Meeting, Orlando, FL, January 2010.

[13] Post, D., "A new DoD initiative: the Computational Research and Engineering Acquisition Tools and Environments (CREATE) program," Journal of Physics, Conference Series 125, 2008.

[14] Wissink, A., "Am Overset Dual-Mesh Solver for Computational Fluid Dynamics," 7th International Conference on Computational Fluid Dynamics, Paper ICCFD7-1206, Hawaii, July 2012.

[15] Wissink, A., Kamkar, S., Pulliam, T., Sitaraman, J., and Sankaran, V., "Cartesian Adaptive Mesh Refinement for Rotorcraft Wake Resolution," AIAA-2010-4554, 28th Applied Aerodynamics Conference, Chicago, IL, June 2010.

[16] Sitaraman, J., Floros, M., Wissink, A., and Potsdam, M., "Parallel domain connectivity algorithm for unsteady flow computations using overlapping and adaptive grids," Journal of Computational Physics, Vol. 229, No. 12, pp. 4703-4723, 2010.

[17] Wissink, A., Sitaraman, J., Sankaran, V., Mavriplis, D., and Pulliam, T., "A Multi-Code Python-Based Infrastructure for Overset CFD with Adaptive Cartesian Grids", AIAA-2008-0927, 46th AIAA Aerosciences Conference, Reno, NV, January 2008.

[18] Sitaraman, J., Katz, A., Jayaraman, B., Wissink, A., and Sankaran, V., "Evaluation of a Multi-Solver Paradigm for CFD using Unstructured and Structured Adaptive Cartesian Grids," AIAA-2008-0660, 46th AIAA Aerosciences Conference, Reno, NV, January 2008.

[19] Spalart, P., and Allmaras, S., “A one-equation turbulence model for Aerodynamic flows,” Le Recherche Aerospátiale, Vol. 1, pp. 5-21, 1994.

[20] Allmaras, S., Forrester, J., and Spalart, P., "Modifications and Clarifications for the Implementation of the Spalart-Allmaras Turbulence Model," ICCFD-1902, 7th International Conference on Computational Fluid Dynamics (ICCFD7), Big Island, Hawaii, July 2012.

[21] Roe, P.L., “Approximate Riemann Solvers, Parameter vectors, and Difference Schemes," Journal of Computational Physics, Vol. 43, pp. 357$372,1981$.

[22] Thomas, P., and Lombard, C., "Geometric Conservation Law and Its Application to Flow Computations on Moving Grids," AIAA Journal, Vol. 17, No. 10, pp. 1030-1037, 1979.

[23] Van Albada, G., Van Leer, B., and Roberts, W., "A comparative study of computational methods in cosmic gas dynamics," Astronomy and Astrophysics Vol. 108, pp. 76-84, 1982.

[24] Koren, B., "Upwind Schemes, Multigrid and Defect Correction for the Steady Navier-Stokes Equations," Proceedings of the 11th International Conference on Numerical Methods in Fluid Dynamics, Willamsburg, VA, June 1988.

[25] Venkatakrishnan, V., "On the Accuracy of Limiters and Convergence to Steady-State Solutions," AIAA-1993-0880, 31st AIAA Aerospace Sciences Meeting and Exhibit, Reno, NV, January 1993.

[26] Mavriplis, D., "Revisiting the Least-Squares Procedure for Gradient Reconstruction on Unstructured Meshes," AIAA-2003-3986, 16th AIAA Computational Fluid Dynamics Conference, Orlando, FL, June 2003.

[27] Saad, Y., and Schultz, M., "GMRES: a generalized minimal residual algorithm for solving nonsymmetric linear systems,” SIAM Journal on Scientific and Statistical Computing, Vol. 7, No. 3, pp. 856-869, 1986.

[28] Karypis, G. and Kumar, V., "A Fast and Highly Quality Multilevel Scheme for Partitioning Irregular Graphs," SIAM Journal on Scientific 
Computing, Vol. 20, No. 1, pp. 359-392, 1999.

[29] Berger, M., and Colella, P., “Local Adaptive Mesh Refinement for Shock Hydrodynamics," Journal of Computational Physics, Vol. 82, pp. 65-84, 1989.

[30] Kamkar, S., Wissink, A., Sankaran, V., and Jameson, A., "Feature-Driven Cartesian Adaptive Mesh Refinement for Vortex-Dominated Flows," Journal of Computational Physics, Vol. 230, No. 16, July 2011, pp. 6271-6298.

[31] Johnson, T, and Patel, V. "Flow past a sphere up to a Reynolds number of 300.” Journal of Fluid Mechanics, Vol. 378, pp. 19-70, 1999.

[32] Salari, K., and Knupp, P. "Code Verification by the Method of Manufactured Solutions," Sandia Report, Sandia National Laboratories, 2000.

[33] Roache, P., "Code Verification by the Method of Manufactured Solutions," Transactions of the ASME, Vol. 124, pp. 4-10, 2002.

[34] Wissink, M., Sitaraman, J., Katz, A., and Roget, B. “Application of 3D Strand Mesh Technology to Rotorcraft Hover," AIAA-2015-0044, 53rd AIAA Aerospace Sciences Meeting, Kissimmee, FL, January 2015.

[35] National Aeronautics and Space Adiminstration. “Turbulence Modeling Resource,” NASA Langley, 2012. http://turbmodels.larc.nasa.gov/.

[36] Ladson, C., "Effects of Independent Variation of Mach and Reynolds Numbers on the Low-Speed Aerodynamic Characteristics of the NACA 0012 Airfoil Section," NASA TM 4074, October 1988.

[37] Schmitt, V., and Charpin, F., "Pressure Distributions on the ONERA-M6-Wing at Transonic Mach Numbers," Experimental Data Base for Computer Program Assessment, Report of the Fluid Dynamics Panel Working Group 04, AGARD AR 138, May 1979.

[38] Martin, P., Pugliese, G., and Leishman, J., "High Resolution Trailing Vortex Measurements in the Wake of a Hovering Rotor," Journal of the American Helicopter Society, Vol. 49, No. 1, pp. 39-52, 2003.

[39] Duraisamy, K., Ramasamy, M., Baeder, J., and Leishman, J., "High-Resolution Computational and Experimental Study of Rotary-Wing Tip Vortex Formation,” AIAA Journal, Vol. 45, No. 11, pp. 2593-2602, 2007.

[40] Johnson, W., "Calculation of Tilt Rotor Aeroacoustic Model (TRAM DNW) Performance, Airloads, and Structural Loads," AHS Aeromechanics Specialists' Conference, Atlanta, GA, November 2000.

[41] Mavriplis, D., and Mani, K., "Unstructured Mesh Solution Techniques using the NSU3D Solver," AIAA Paper 2014-0081, 52nd AIAA Aerospace Sciences Conference, National Harbor, MD, January 2014.

[42] Wissink, A., "Investigation of Dual Mesh CFD Approach for Hover," 5th Decennial AHS Aeromechanics Specialists' Conference, San Francisco, CA, January 2014.

[43] Buning, P., et. al. “OVERFLOW Users Manual,” NASA Langley Research Center, July 2003. 